\title{
Stichwort: Lernzentriertes Leitungshandeln an Schulen - Leadership for Learning
}

\author{
Pierre Tulowitzki $\mathbb{D} \cdot$ Marcus Pietsch $\mathbb{D}$
}

Eingegangen: 3. Dezember 2019 / Überarbeitet: 27. April 2020 / Angenommen: 11. August 2020 / Online publiziert: 10 . September 2020

(C) Der/die Autor(en) 2020

Zusammenfassung Führung gilt als wichtiger Aspekt effektiver Schulen. Im internationalen Raum hat sich in diesem Zusammenhang in den vergangenen Jahren das Konzept des lernzentrierten Leitungshandelns an Schulen, Leadership for Learning, etabliert. Leadership for Learning wird hier stets ergebnisorientiert gedacht und rückt den Kompetenzerwerb von Schüler*innen in den Fokus des Leitungshandelns. Lernzentriertes Leitungshandeln zielt dabei nicht ausschließlich auf die Unterrichtsebene ab, auch das Lernen von Lehrkräften und das Organisationale Lernen sollen, im Sinne eines Capacity Building, gefördert werden. Entsprechend geht der Ansatz über klassische Annahmen zur unterrichtsbezogenen Führung (Instructional Leadership) hinaus, indem es deren Einschränkungen, nämlich eine Verengung der Perspektive auf die Person der Schulleitung sowie den Unterricht, überwindet. Im deutschsprachigen Raum ist dieses Konzept, obwohl z. B. in internationalen Schulvergleichsuntersuchungen genutzt, anders als in den anglophonen Ländern, bislang kaum bekannt. Daher werden im vorliegenden Stichwortbeitrag das Konzept des Lernzentrierten Leitungshandelns sowie dessen theoretische Grundannahmen vorgestellt. Darüber hinaus werden Forschungsbefunde zum Thema berichtet und abschließend eine Agenda für die zukünftige Forschung zum Leadership for Learning im deutschsprachigen Raum erarbeitet.

Schlüsselwörter Führung · Leadership for Learning · Lernen · Schulleitung · Schülerleistung

Prof. Dr. P. Tulowitzki

Fachhochschule Nordwestschweiz FHNW, Bahnhofstrasse 6, 5210 Windisch, Schweiz

E-Mail: pierre.tulowitzki@fhnw.ch

PD Dr. M. Pietsch $(\bowtie)$

Leuphana Universität Lüneburg, Universitätsallee 1, 21335 Lüneburg, Deutschland

E-Mail: marcus.pietsch@leuphana.de

Universität Zürich, Freiestrasse 36, 8032 Zürich, Schweiz 


\title{
Learning-centered Leadership_Leadership for Learning
}

\begin{abstract}
Leadership is regarded a crucial aspect of effective schools. Internationally, the concept of Leadership for Learning has received significant attention over the past years. Leadership for Learning is viewed as being focused on outcomes and puts the students' acquisition of competencies in the center of all efforts. Leadership for Learning does not only aim at the level of classroom instruction, but the learning of teachers as well as organizational learning in the sense of capacity building. Consequently, this approach goes beyond classic leadership theories (i.e., instructional leadership), overcoming perspectives focused mostly on the principal or the classroom. However, although it is used in international large-scale comparative studies, the concept of Leadership for Learning is so far fairly unknown in the German-speaking area, as opposed to the anglophone countries. Thus, the concept of Leadership for Learning and its theoretical foundation are introduced in this article. Furthermore, findings of research on Leadership for Learning are reported. Finally, an agenda regarding future research on Leadership for Learning in the Germanspeaking area is developed.
\end{abstract}

Keywords Leadership · Leadership for Learning · Learning · School leadership · Student achievement

\section{Einleitung}

Schulleitungen wird - nicht nur international, sondern auch im deutschen Kontext eine zentrale Rolle in Bezug auf Schulorganisation, -qualität und -entwicklung und ein vermittelter Effekt auf Schüler*innenleistungen zugeschrieben (aus Deutschland u. a. Bonsen et al. 2002; Pietsch und Tulowitzki 2017; international u. a. Pont et al. 2008; Robinson et al. 2009; Leithwood und Louis 2012). Insbesondere Führung bzw. Leadership gilt dabei als besonders relevanter Aspekt effektiver, lernwirksamer Schulen (Mulford 2003; Day et al. 2016). Unter Führung verstehen wir die durch den Kontext geprägte, zielgerichtete soziale Einflussnahme schulischen Personals auf andere Personen (bzw. weiteres Personal) im System Schule (vgl. Wegge und von Rosenstiel 2004).

Während lange Zeit einzelne theoretische Konzepte und Modelle wie z. B. instruktionale und transformationale Führung (Hallinger 2003) den fachwissenschaftlichen Diskurs bestimmten, hat sich in den vergangenen Jahren auf internationaler Ebene zunehmend das Konzept des lernzentrierten Leitungshandelns, des Leadership for Learning (LFL), etabliert. Leadership for Learning bezieht sich auf Leitungshandeln, ist - im Gegensatz zu älteren Konzeptionen - jedoch nicht auf die Schulleitung beschränkt. Trotz einer deutlichen Präsenz in wissenschaftlichen Publikationen (Boyce und Bowers 2018a; Moral et al. 2018; OECD 2016; Bowers 2020) lassen sich Bezüge auf lernzentriertes Leitungshandeln in der Schule in praxisorientierten Publikationen eher selten finden.

Im deutschsprachigen Raum hat dieses Modell darüber hinaus bislang eher wenig Aufmerksamkeit erfahren. Einschränkend ist diesbezüglich zu erwähnen, dass unklar 
ist, ob und inwiefern anglophon geprägte Führungsmodelle, die in einem Kontext mit ausgeprägten Mechanismen der Rechenschaftslegung (high accountability) und vergleichsweiser großer Autonomie und Ressourcenausstattung der Schulleitung situiert sind, in einem Kontext wie Deutschland, der von hoher Autonomie der Lehrkräfte und eher geringer Rechenschaftspflicht der Einzelschule (low accountability) geprägt ist, anwendbar sind (Klein 2016a). So gibt es deutliche empirische Hinweise darauf, dass das Amt der Schulleitung in Deutschland - trotz einer zunehmenden Betonung von Führungshandeln in den mit dieser Stelle verbundenen Aufgabenprofilen - primär durch administrative Tätigkeiten geprägt ist (Huber et al. 2013; Brauckmann und Schwarz 2015). Doch erscheint das Modell des lernzentrierten Leitungshandelns auch für den hiesigen Kontext relevant, da es im Gegensatz zu rein unterrichtsbezogener Führung (instructional leadership) durch eine ganzheitlichere Perspektive wie auch die Betonung von Partizipationsmöglichkeiten eine deutliche Nähe zu Leadership-Strategien aufweist, die an deutschen Schulen identifiziert wurden (Klein 2016b). Im vorliegenden Beitrag soll Leadership for Learning inklusive der zentralen Annahmen, auf denen es aufbaut, vorgestellt, erörtert und im deutschen wie auch internationalen Diskurs rund um Schulentwicklung und schulische Führung verortet werden. Darüber hinaus werden Forschungsbefunde präsentiert und Desiderata für Forschung und Praxis herausgearbeitet.

\section{Theoretischer Zugang zum lernzentrierten Leitungshandeln}

Lernzentriertes Leitungshandeln an Schulen bzw. Leadership for Learning wird seit einigen Jahren vor allem in den westlichen anglophonen Ländern (u. a. Vereinigtes Königreich, USA, Kanada, Australien) diskutiert, ist inzwischen jedoch auch darüber hinaus bekannt. Bisher hat sich keine einheitliche Definition durchgesetzt. Für den Diskurs prägend waren unter anderem die Arbeiten rund um den britischen Educational-Leadership-Forscher John MacBeath: In einem internationalen Forschungsprojekt erarbeitete er gemeinsam mit Kolleg*innen folgende Prinzipien als konstituierende Merkmale lernzentrierten Leitungshandelns: (1) Einen Fokus auf das Lernen, (2) eine lernförderliche Umgebung, (3) Austausch über das Lernen, (4) geteilte Führungsverantwortung und (5) eine nach innen wie nach außen hin aktive Kultur der Verantwortung (Townsend und MacBeath 2011, Kapitel 1). Die Prinzipien beziehen sich dabei explizit auf alle Mitglieder der Schulgemeinschaft (Schüler*innen, Lehrkräfte, Schulleiter*innen, MacBeath 2007).

Leadership for Learning ist in der Regel ergebnisorientiert, rückt den Kompetenzerwerb von Schüler*innen in den Fokus des Leitungshandelns (Hallinger 2011) und greift dabei den aktuellen Diskurs zu effektivem Leitungshandeln aus verschiedenen Bereichen auf (Daniëls et al. 2019; siehe Abb. 1).

Lernzentriertes Leitungshandeln zielt dabei nicht ausschließlich auf die Unterrichtsebene ab, auch das Lernen der Lehrkräfte und schließlich auch das Lernen auf organisationaler Ebene sollen gefördert werden (Hallinger und Heck 2010b). So ist das zentrale Ziel zwar das (verbesserte) Lernen von Schüler*innen, dies aber vermittelt über das Lernen auf allen Ebenen des Systems Schule - die Organisation, die Lehr- sowie die Leitungskräfte (Townsend et al. 2013). Folglich wird lernzentriertes 


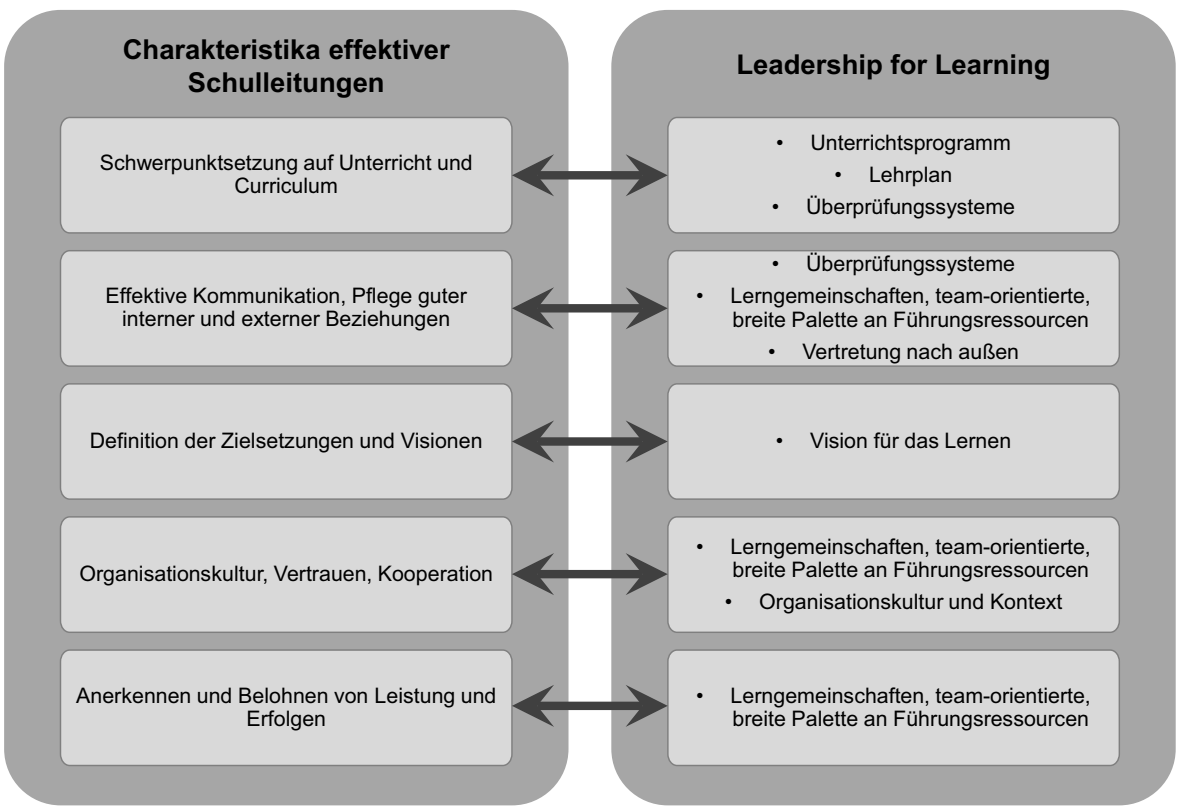

Abb. 1 Beziehungen zwischen Leadership for Learning und Charakteristika effektiver Schulleitungen (basierend auf Daniëls et al. 2019, S. 121, sowie auf Murphy et al. 2007)

Leitungshandeln als etwas betrachtet, das Sache der gesamten Schulgemeinschaft ist. Den Beziehungen zwischen schulischen Akteuren wird eine besondere Bedeutung beigemessen, da diese als Voraussetzungen angesehen werden, damit relevantes Wissen, das Unterricht und Lernchancen verbessern kann, in der Organisation Schule entwickelt und allen Schulbeteiligten zur Verfügung gestellt werden kann (Timperley und Robertson 2011).

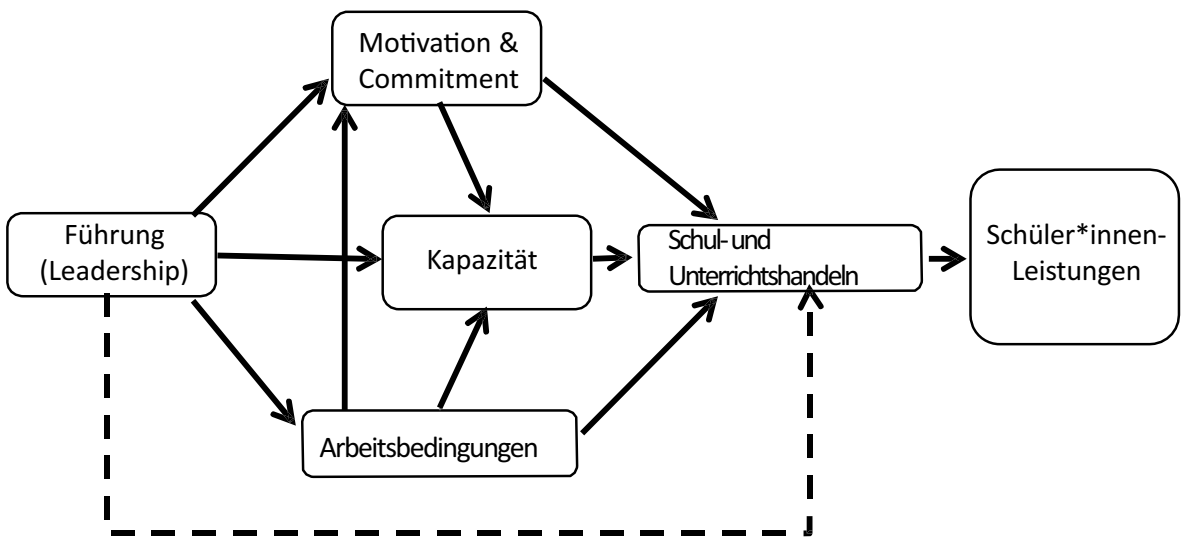

Abb. 2 Wirkungen von Leitungshandeln auf innerorganisatorische Faktoren, Schul- und Unterrichtshandeln sowie Schüler*innenleistung (nach Leithwood und Jantzi 2006, S. 204, Übersetzung durch die Autoren) 
Das Modell zu Effekten von Führung auf Schüler*innenleistung von Leithwood (Leithwood et al. 2004; Leithwood und Jantzi 2006; Louis et al. 2010a, Abb. 2) verdeutlicht diese Annahme: Führungshandeln beeinflusst demnach die Arbeitsbedingungen sowie die Kapazität und Motivation der Mitarbeitenden in der Schule. Diese Variablen beeinflussen wiederum das Unterrichtshandeln, welches wiederum einen Einfluss auf die Leistungen von Schüler*innen hat. Führungshandeln wird somit als indirekt wirksam in Bezug auf die Leistungen von Schüler*innen erachtet.

Im Kern handelt es sich beim Ansatz des lernzentrierten Leitungshandelns um ein erweitertes Verständnis instruktionaler Führung, das jedoch nicht mehr ausschließlich auf die Person(en) der (formalen) Schulleitung sowie den Unterricht fokussiert (Bush und Glover 2014). Nach Murphy et al. (2007) umfasst lernzentriertes Leitungshandeln folgende Elemente: Fokus auf Lernen, Unterrichten und die Lerninhalte sowie Bemühungen, alle anderen Bereiche der Schule (Verwaltung, Organisation, Finanzen) auf die Unterstützung der erstgenannten Elemente hin auszurichten. Hallinger (2011) versteht lernzentriertes Leitungshandeln daher als eine Triade von (traditionellen) Führungsstilen, die Elemente transformationaler und unterrichtsbezogener Führung beinhaltet, aber auch eine starke Orientierung hin zu ge- bzw. verteilter Führung aufweist (Hallinger 2011; siehe auch OECD 2016, Kapitel 2).

In diesem Verständnis rückt die einzelne (formale) Schulleitung als Person etwas in den Hintergrund. Demgegenüber werden kollaborative Elemente wie ein ständiger Dialog zwischen den Schulbeteiligten über die pädagogischen Ziele und wie diese im Schulalltag durch verantwortungsbewusstes Handeln gemeinsam erreicht werden können stärker betont. Entsprechend müssen im Zuge eines derartigen Verständnisses von Führung die Lernziele aller Schulbeteiligten (nicht nur der Schüler*innen) sowie der Organisation Schule, die sich als Reaktion auf dynamische Kontexte stetig wandeln können, immer wieder neu ausgehandelt, das Handeln der einzelnen Akteure hierauf abgestimmt und die Zielerreichung kontinuierlich überprüft werden. Statt eine klare Hierarchisierung zwischen Führenden und Geführten vorzunehmen, wird Führungshandeln als eine Aktivität verstanden, die gemeinsam von Führenden und Beteiligten konstituiert bzw. ko-konstruiert wird. Entsprechend berücksichtigt Leadership for Learning auch die für das Unterrichtsgeschehen relevante Dimension des Teacher Leadership (Supovitz et al. 2010):

Leadership for learning is a distinct form of educational practice that involves an explicit dialogue, maintaining a focus on learning, attending to the conditions that favour learning, and leadership that is both shared and accountable. Learning and leadership are conceived of as ,,activities“ linked by the centrality of human agency within a framework of moral purpose (Swaffield und MacBeath 2009, S. 42).

Daniëls et al. (2019) zeigen in einem Review zum lernzentrierten Leitungshandeln an Schulen entsprechend, dass sich das Konzept aus vier unterschiedlichen theoretischen Ansätzen speist (s. Abb. 3): 1) Der unterrichtszentrierten Führung (instructional leadership), 2) der transformationalen Führung (transformational leadership), 3) Praktiken der geteilten bzw. verteilten Führung (shared bzw. distributed leadership) und 4) Ansätzen der situativen Führung. 


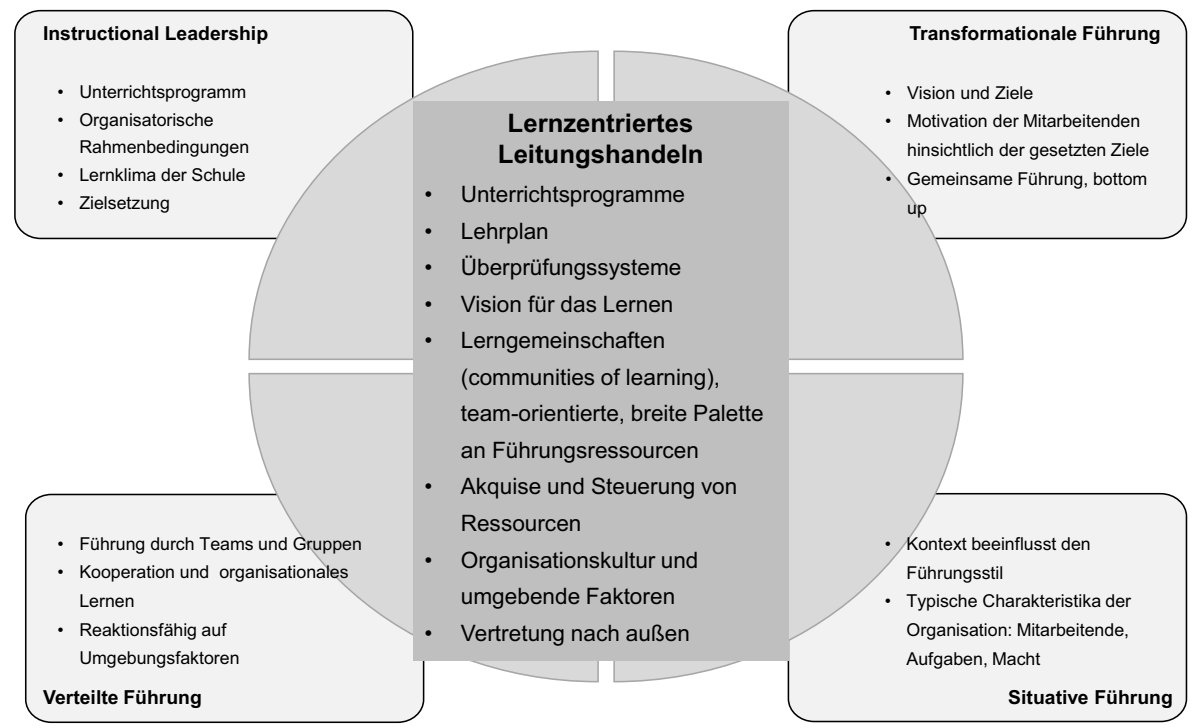

Abb. 3 Beziehungen zwischen instruktionaler, transformationaler, verteilter und situativer Führung sowie lernzentriertem Leitungshandeln (basierend auf Daniëls et al. 2019, S. 117)

Aufgrund ihrer zentralen Bedeutung als historische Vorläufer und Teilkomponenten des lernzentrierten Leitungshandeln werden die Ansätze nachfolgend kurz vorgestellt.

\subsection{Unterrichtsbezogene Führung (Instructional Leadership)}

Bei der instruktionalen oder unterrichtsbezogenen Führung (instructional leadership) wird die Aufmerksamkeit auf den direkten Einfluss der Schulleitung rund um die Verbesserung von Lehr-Lernprozessen gelegt; somit stehen Belange der Unterrichtsentwicklung oftmals im Fokus (Leithwood et al. 1999). Insbesondere Unterrichtspraktiken von Lehrkräften bzw. dem Unterrichtsgeschehen wird besondere Beachtung geschenkt. Schulleitungen, die diesem Modell folgen, prägen und gestalten Unterricht und Erziehung an den durch sie geleiteten Schulen aktiv. Sie stellen hohe Ansprüche an Lern- und Entwicklungsziele, stimmen z. B. curriculare Ziele mit tatsächlichen Lehr- und Lernpraktiken an der Schule ab, richten individuelle Fortbildungsmaßnahmen an diesen Zielen aus, supervidieren Unterricht und Lernfortschritte, geben evidenzbasierte Rückmeldungen zum Lernen und Lehren und sind kompetente Ansprechpartner*innen, wenn Probleme in Unterrichtsfragen auftreten. Somit wird vorausgesetzt, dass die Schulleitung besonders kompetent in Bezug auf Lehr-Lernprozesse ist, aber auch in Bezug auf Lehrpläne versiert genug ist, um diesbezüglich Entscheidungen zu treffen und auf Veränderungen hin einzuwirken.

Ergebnisse einer Meta-Evaluation mit Daten aus dem größtenteils anglophonen Raum, deuten darauf hin, dass Schulleitende, die solch instruktionale Führungspraktiken häufig nutzen, durch ihr Handeln den Schüler*innen an den durch sie geleiteten Schulen einen zusätzlichen Lernvorsprung von rund anderthalb bis zwei 
Lernjahren ermöglichen können (Robinson et al. 2008; vgl. hierzu auch Hattie 2009). Auch wenn sie im deutschsprachigen Kontext eher wenig verbreitet zu sein scheint (Feldhoff et al. 2008; Harazd 2010), sehen manche Autor*innen Anzeichen für eine langsame Orientierung des deutschen Bildungssystems hin zu unterrichtsbezogener Führung (Brauckmann et al. 2016).

\subsection{Transformationale Führung (transformational leadership)}

Transformationale Führung beschäftigt sich mit einer grundsätzlichen Kultur der Innovation und Veränderung in einer Organisation. Der Begriff wird in der Regel auf den US-Politologen und Historiker James MacGregor Burns zurückgeführt, der anhand einer historischen Analyse rund um Führung eine Unterscheidung zwischen transaktionaler und transformierender Führung vornahm (Burns 1978). Transformierende Führung ereignete sich laut Burns dann, wenn leitende Personen so mit anderen interagierten, dass sich beide gegenseitig motivieren und höheren Leistungen wie auch moralisch höheren Standards anspornen (Burns 1978, S. 20). Dieses Verständnis wurde von Bernard Bass unter dem Begriff der transformationalen Führung erweitert. Bass zufolge erfolgt transformationale Führung wenn Führungskräfte die Interessen und Aufmerksamkeit der Angestellten so erweitern, dass diese nicht (mehr) ihre eigenen Interessen, sondern die der Gruppe priorisieren und ein Verständnis für „das große Ganze“ entwickeln (Bass 1990, S. 21). In der Regel bedienen sich Leitungskräfte Bass zufolge transaktionaler als auch transformationaler Führung.

Transformationale Führung lässt sich dabei in folgende Dimensionen unterteilen und von den anderen Dimensionen des LfL - unterrichtsbezogene und geteilte Führung - abgrenzen (Bass und Avolio 1993; Leithwood und Jantzi 2009; Scheerens 2012):

1. Idealized Influence (Vorbildcharakter und Glaubwürdigkeit)

2. Inspirational Motivation (Motivation durch begeisternde Visionen)

3. Intellectual Stimulation (Anregung und Förderung von kreativem Denken)

4. Individualized Consideration (Individuelle Unterstützung und Förderung)

Transformationaler Führung im schulischen Kontext zielt häufig darauf ab, diese in ihrer Gesamtheit aktiv und nachhaltig weiter zu entwickeln, sie in lernende Organisationen zu verwandeln und es dem schulischen Personal zu ermöglichen, kritisch-konstruktiv mit Innovationen und Veränderungen umzugehen.

Während es empirisch gestützte Hinweise gibt, dass sich eine transformationale Führung in der Schule positiv auf die Innovationskapazität der Lehrkräfte auswirkt (Leithwood und Jantzi 2005, 2006), wird der Effekt auf die Leistungen von Schüler*innen als schwächer als jener unterrichtsbezogener Führung erachtet (Robinson et al. 2008; Shatzer et al. 2014). Eine in diesem Zusammenhang gelegentlich formulierte Zuspitzung lautet, dass transformationale Führung primär auf Lehrkräfte/ Mitarbeitende und unterrichtsbezogene primär auf Schüler*innen abziele (vgl. Hattie 2015). Transformationale Führung von Schulleitungen kann sich positiv auf die Arbeitszufriedenheit von Lehrkräften auswirken, das Risiko für einen Burnout und 
in Folge dessen die Anzahl krankheitsbedingter Fehltage pro Schuljahr um etwa sechs bis acht Prozent senken (vgl. Harazd und Ophuysen 2011).

\subsection{Partizipative Führung (shared bzw. distributed leadership)}

Partizipative Führung ist darauf ausgerichtet, Mitarbeiter*innen in Entscheidungsprozesse einzubeziehen und Führungsverantwortung auf verschiedene Beteiligte innerhalb einer Organisation zu verteilen. Ziel eines solchen Führungshandelns ist es, eine Kultur zu schaffen, in deren Rahmen sich die Mitarbeiter*innen einer Schule aktiv in die Ausgestaltung der Führung einbringen können. Auf diesem Wege sollen innerschulische Kohärenz hergestellt, die Bereitschaft zur Verantwortungsübernahme von Einzelpersonen gesteigert und letztlich die Effektivität des Steuerungshandelns erhöht werden. Modelle partizipativer Führung repräsentieren die Abkehr einer individuellen, „,heroischen“ Führungskraft zugunsten eines Verständnisses von Führung, in welchem mehr Augenmerk auf Teamstrukturen, Zusammenarbeit sowie Leitungshandeln aller Mitglieder im System Schule (Schulleitende, Lehrkräfte, weitere Mitglieder, Schüler*innen) legt (vgl. Harris 2004; Harris und Spillane 2008).

Praktiken geteilter Führung (shared leadership) zielen darauf ab, Partizipation von Lehrkräften in den schulischen Entscheidungsprozessen zu ermöglichen und zu fördern. Dies betrifft sowohl die informelle als auch die formalisierte Ebene (Printy und Marks 2006). Das Modell der verteilten Führung (distributed leadership) stellt individuelle Führungskräfte und Mitarbeitende hingegen eher in den Hintergrund und legt den Schwerpunkt stattdessen auf die Führungspraktiken an sich. Somit kann jede Person als leitend erachtet werden, welche (und sei es nur kurzzeitig) „Aktivitäten welche zu den Kernfunktionen der Organisation in Verbindung stehen und dazu dienen, die Motivation, die Wissensbasis oder das Handlungsrepertoire der anderen Mitglieder der Organisation zu beeinflussen“ (Spillane 2006, S. 11 f., Übersetzung durch die Autoren). Beiden Perspektiven (geteilte und verteilte Führung) ist gemein, dass der Blick auf Führungskonstellationen dynamisch ist - wer in einer Situation leitet, mag in einer anderen geleitet werden.

Schulen, an denen solche Prinzipien genutzt werden, sind mit Blick auf die Qualität von Schule und Unterricht sowie Schüler*innenleistungen langfristig vor allem dann erfolgreich, wenn die Verteilung der Führung formalisiert wurde, also Pläne und Absprachen vorliegen, aus denen hervorgeht, welche Personen oder Personengruppen für bestimmte Entscheidungen verantwortlich sind (Harris 2008). Dabei wirkt sich die Verteilung von Führung insbesondere auf die Schul- und Unterrichtsentwicklung (Harris 2008), die Identifikation von Lehrkräften mit ihren Schulen (Hulpia et al. 2009) sowie vermittelt über diese Faktoren auf die Lernentwicklung von Schüler*innen (vgl. Leithwood und Mascall 2008) aus. Internationale empirische Arbeiten deuten auf einen indirekten und (kleinen) positiven Zusammenhang zwischen verteilter Führung und Schüler*innenleistung hin (Tian et al. 2016).

\subsection{Situative Führung und Kontext}

Kern des Konzepts der kontingenten oder situativen Führung ist die Annahme, dass erfolgreiches Führungshandeln von einer Vielzahl von Faktoren abhängig ist. Es 
kommt also nicht nur auf das Verhalten einer Führungskraft, sondern auch auf Aspekte wie die Beziehung zu den Mitarbeitenden, Machtverhältnisse, Organisations- und Tätigkeitsstrukturen, bisherige (prägende) Entwicklungen und weiteren Rahmenbedingungen an.

Situative Führungsmodelle erlebten in der Managementlehre in den 1970er und 1980er Jahren eine Phase der Popularität; zentral war hier das Modell der situativen Führung von Hersey und Blanchard (1982), welches unterschiedliche (optimale) Verhaltensweisen für Führungskräfte, je nach „Reifegrad“ (maturity level) der Mitarbeitenden definiert. In der Schulleitungs- und Schulentwicklungsforschung erschienen im anglophonen Raum zahlreiche Arbeiten, die auf diesen Konzeptionen aufbauten (u. a. Vecchio 1987; Kelley et al. 2005; Thompson und Glasø 2015).

Während situative Führung als dediziertes Führungsmodell im heutigen schulischen Führungsdiskurs eine eher untergeordnete Rolle spielt, so wird dem Kontext durchaus eine bedeutsame Rolle zugesprochen. Kontext wird dabei als relevanter Faktor für erfolgreiches Führungshandeln an Schulen erachtet (Opdenakker und Damme 2007; Leithwood et al. 2020, Abschn. 3). Führungshandeln wird als eingebettet in den institutionellen, unmittelbaren räumlichen Kontext (im Englischen: „community context“, siehe hierzu auch Forschung zur „Area close to school“, Schwarz und Brauckmann 2015), einem sozio-kulturellen Kontext, einem politischen und ökonomischen Kontext sowie einem Schulentwicklungskontext, der auch als einzelschulhistorischer Kontext bezeichnet werden könnte, betrachtet (Hallinger 2016). Abb. 4 veranschaulicht, wie diese Kontexte Führungshandeln beeinflussen können. Hallinger (2016) warnt als Konsequenz davor, dass gerade in großflächigen Studien durch das Konzentrieren auf Mittelwerte mögliche starke Kontextfaktoren

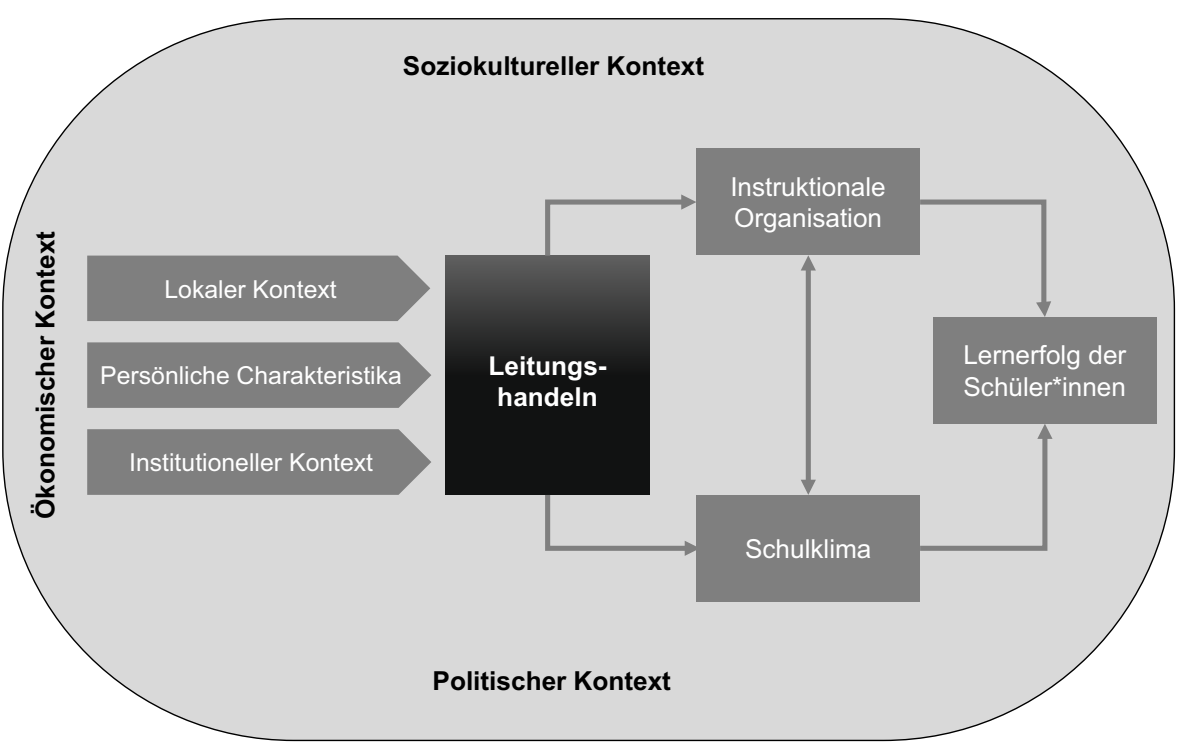

Abb. 4 Kontextfaktoren rund um Führung in der Schule (nach Hallinger 2016, S. 17) 
,in den Schatten versteckt“ blieben (Hallinger 2016, S. 16), ihre Relevanz also verkannt würde.

\section{Empirische Forschung zum lernzentrierten Leitungshandeln}

Obwohl das LFL-Modell auf internationaler Ebene seit geraumer Zeit diskutiert wird und sich aus dem Konzept der instruktionalen Führung entwickelt hat (Hallinger 2011; Boyce und Bowers 2018b; Daniëls et al. 2019), liegen bislang nur wenige empirische Befunde vor, die den theoretischen Annahmen der zugrunde liegenden Modelle in vollem Umfang gerecht werden. Insbesondere aus dem deutschsprachigen Raum wurden entsprechende Befunde bislang kaum berichtet. So zeigen Tulowitzki et al. (2020), dass beispielsweise das Verhältnis von empirischen Untersuchungen, die sich mit dem Zusammengang lernzentrierten Leitungshandelns und innerschulischen Humanressourcen (z. B. Arbeitszufriedenheit oder Commitment von Lehrkräften) beschäftigen, im Vergleich zwischen den USA und Deutschland bei $14 \mathrm{zu}$ eins liegt. Aber auch international finden sich nur wenige Untersuchungen, die der Komplexität des Ansatzes Rechnung tragen und über die Analyse einzelner Teilaspekte hinausgehen. Dies mag vor allem daran liegen, dass sich, infolge der Komplexität der Annahmen, die dem Konzept des lernzentrierten Leitungshandelns zugrunde liegen, bei dessen Erforschung eine Vielzahl verschiedenere Herausforderungen an die Konzeption, Durchführung und Auswertung entsprechender Studien stellen.

So wird erstens angenommen, dass es sich bei dem Konzept um ein mehrdimensionales Konstrukt handelt, das verschiedene Facetten von Führungshandeln umfasst, die wiederum auf unterschiedlichen theoretischen Annahmen und Traditionen gründen. Zweitens wird erwartet, dass Leitungshandeln auf verschiedenen Ebenen des Systems Schule stattfinden kann, z. B. zwischen einer Schulleitung und dem Lehrerkollegium, der Schulleitung und Gruppen von Lehrkräften, der Schulleitung und einzelnen Lehrkräften, aber auch - dies ist vor allem die Perspektive der geteilten Führung - zwischen Lehrkräften im Kollegium. Drittens ist bekannt und wird erwartet, dass Effekte von Führung zumeist vermittelt, also indirekt zeitigen. Und viertens wird angenommen, dass Lernen als ein (dynamischer und ggf. reziproker) Prozess zu verstehen ist, der im zeitlichen Verlauf zu einer Entwicklung bzw. Veränderung von Kompetenzen, Wissen, Haltungen und den damit einhergehenden Verhaltensweisen und -mustern der Schulbeteiligten führt.

Für das Design entsprechender Studien sowie für die Datenanalyse bedeutet dies jedoch, dass folgende Parameter zu berücksichtigen sind:

1. Lernzentriertes Leitungshandeln als mehrdimensionales Konstrukt

2. Lernzentriertes Leitungshandeln als Mehrebenenkonstrukt

3. Mediationsbeziehung zwischen lernzentriertem Leitungshandeln und Outcome

4. Lernzentriertes Leitungshandeln als Intervention, die zu Veränderungen führt 


\subsection{Lernzentriertes Leitungshandeln als mehrdimensionales Konstrukt}

Lernzentriertes Leitungshandeln umfasst Aspekte instruktionaler, transformationaler, geteilter und situationsbezogener Führung (Daniëls et al. 2019). Entsprechend handelt es sich hierbei um ein theoretisches Konzept, das sich auf distinkte, jedoch untereinander zusammenhängende Dimensionen bezieht und das empirisch mithilfe mehrdimensionaler Konstrukte erfasst werden muss (Law et al. 1998). Obwohl in einer Vielzahl von Studien angegeben wird, dass dort lernzentriertes Leitungshandeln untersucht wird, werden zumeist nur einzelne Aspekte bzw. Dimensionen herausgegriffen, zumeist entweder der Aspekt der instruktionalen Führung oder der geteilten, partizipativen Führung. So messen Instrumente, wie das US-amerikanische Schools and Staffing Survey (SASS) oder das Vanderbilt Assessment of Leadership in Education (VAL-ED), die vielfach für Untersuchungen zum Thema herangezogen werden, vor allem den Aspekt der instruktionalen Führung (Porter et al. 2010; Goldring et al. 2015; Boyce und Bowers 2018a, 2018b). Designs bzw. Instrumente, die beanspruchen möglichst alle relevanten Dimensionen lernzentrierten Leitungshandelns zu erfassen, liegen hingegen kaum vor.

Ein US-amerikanisches Instrument, das ausdrücklich dafür entworfen wurde lernzentriertes Leitungshandeln an Schulen im Sinne der theoretischen Konzeptualisierung zu erfassen, ist das Comprehensive Assessment of Leadership for Learning (CALL, Blitz et al. 2014). Das Instrument wurde für formative Assessment- und Feedbackzwecke entwickelt und soll sowohl Schulleitungen als auch Lehrkräfte dabei unterstützen ihre instruktionalen, transformationalen und geteilten Führungskompetenzen auszubauen und hierdurch das Lehren bzw. den Unterricht an der eigenen Schule weiter zu entwickeln. Befragt werden sowohl Schulleitungen als auch Lehrkräfte. Dabei liegt der Fokus auf der Idee einer geteilten-instruktionalen Führung; entsprechend wurden Skalen zur Messung instruktionaler Führung sowie verteilter bzw. geteilter Führung entwickelt, eine explizite Skala zur Messung transformationaler Führung ist nicht vorhanden, wenngleich dieser Aspekt implizit, im Rahmen anderer Skalen des Instruments, miterfasst wird.

International hat sich darüber hinaus das Leadership for Learning-Konzept der OECD (2016) verbreitet. Im Rahmen des Teaching and Learning International Survey (TALIS) und des Programme for International Student Assessment (PISA) wird lernzentriertes Leitungshandeln als Mischung instruktionaler und geteilter bzw. verteilter Führungspraktiken verstanden (OECD 2016), wobei letzter Aspekt insbesondere auf die Partizipation an Steuerungsentscheidungen fokussiert (Beispielitem: „This school provides staff with opportunities to actively participate in school decisions."). Die Skalen zur instruktionalen Führung basieren dabei grosso modo auf der Principal Instructional Management Rating Scale (PIMRS, Hallinger 1994), wobei jedoch nur zwei der drei dort vorhanden Facetten berücksichtigt werden: Schulzielund Unterrichtsmanagement (Lee et al. 2012). Die dritte Facette des Instruments, bei der es darum geht, ob die Schulleitung ein positives Lernklima schafft, und die sich in weiten Teilen mit Aspekten transformationaler Führung deckt bzw. hoch mit dieser korreliert (Hallinger und Wang 2015), wird hingegen in den OECD-Studien nicht berücksichtigt. Befragt werden, je nach Studie, Schulleitungen und/oder Lehrkräfte. 
Im deutschsprachigen Raum verfolgt die Hamburger Schulinspektion einen ähnlichen Ansatz, wobei, anders als im Rahmen der OECD-Studien sowie des CALL ausschließlich Lehrkräfte befragt werden. Eine mehrperspektivische Befragung, die auch die Sicht der Schulleitung beinhaltet, findet nicht statt. Auch wurde kein eigenes Instrumentarium entwickelt, sondern auf bereits vorhandene Instrumente und Skalen zurückgegriffen (Pietsch et al. 2013). Zum Einsatz kommen einerseits Skalen aus den TALIS-Erhebungen zur instruktionalen Führung (OECD 2009, 2010; Schmich und Schreiner 2010). Andererseits werden, analog zu den OECD-Studien, Fragen zur geteilten Führung an Schulen gestellt, die die Teilhabe des Kollegiums an Steuerungsentscheidungen (Shared Leadership Among Principal and Others) erfassen sollen und von Wahlstrom und Louis (2008) entwickelt wurden. Jedoch geht das Hamburger Design über das der OECD hinaus, indem ergänzend auch die transformationale Führung an Schulen erhoben wird. Genutzt wird hierfür eine Kurzform des Multifactor Leadership Questionnaire (MLQ; Avolio und Bass 1995; Felfe 2006; Harazd und van Ophuysen 2011), so dass ein breites Spektrum transformationaler Führungspraktiken (Full Range of Leadership-Ansatz, Avolio 2003) abgebildet wird.

Nur wenig ist bislang darüber bekannt, wie sich die einzelnen Dimensionen des lernzentrierten Leitungshandelns zueinander verhalten. Obwohl eine Vielzahl von Möglichkeiten - z.B. klassische Korrelationsanalysen, (konfirmatorische) Faktorenanalysen, Mehrdimensionale Item-Response-Modelle (MIRT, Hartig und Höhler 2009) oder Statistiken zur Evaluation der praktischen Relevanz von Subscores (Sinharay 2010) - vorhanden ist, um diskriminante Eigenschaften sowie differentielle Effekte festzustellen, wurden diese bisher kaum genutzt. Die wenigen Befunde zum Thema sind darüber hinaus sehr heterogen. So haben beispielsweise Marks und Printy (2003) in einer vielbeachteten Studie den Zusammenhang von geteilter instruktionaler und transformationaler Führung untersucht und konnten zeigen, dass an Schulen, an denen alle drei Führungsfacetten stark ausgeprägt sind, eine überdurchschnittliche Qualität des Unterrichts sowie hohe Schüler*innenleistungen beobachtet werden können. Gleichwohl wurde der Zusammenhang der einzelnen Führungsdimensionen nicht genauer bzw. ausschließlich grafisch spezifiziert, so dass die psychometrischen Eigenschaften unklar bleiben. Shatzer et al. (2014) wiederum haben untersucht, inwieweit sich differentielle Effekte transformationaler und instruktionaler Führungspraktiken mit Blick auf Schüler*innenleistungen nachweisen lassen und zeigen, dass durch instruktionale Führung $45 \%$ und durch transformationale Führung $25 \%$ in der Variation von Schüler*innenleistungen sowie 27 bzw. $22 \%$ der Varianz in Lernzuwächsen erklären lassen. Wie sich jedoch die einzelnen Führungsdimensionen zueinander verhalten wurde auch in dieser Studie nicht untersucht bzw. berichtet. Hallinger und Wang (2015) berichten weiterhin, dass - trotz des langjährigen Vorhandenseins beider Instrumente - aktuell keinerlei Daten vorlägen, die es belastbar erlauben würden, Analysen zum Vergleich der PIMRS und des MLQ durchzuführen, die einem Peer-Review-Prozess standhalten würden. Louis et al. (2010b) haben hingegen den Zusammenhang instruktionaler und geteilter Führung untersucht und berichten anhand eines Strukturgleichungsmodells einen statistisch nicht signifikanten, korrelativen Zusammenhang dieser beiden Führungsdimensionen von $r=0,10$, wobei für beide Dimensionen ein eigenständiger Zusammenhang mit Schüler*innenleistungen festgestellt wurde. Für Deutschland berichten Pietsch 
et al. (2019b) im Gegensatz dazu anhand von Daten der Schulinspektion Hamburg, dass die (latenten) Korrelationen einzelner Facetten des lernzentrierten Leitungshandelns zwischen $r=0,52$ und $r=0,90$ liegen und zeigen mithilfe konfirmatorischer Faktorenanalysen, dass es methodisch sinnvoll ist instruktionale, transformationale und geteilte Führung - und sogar deren Subfacetten - als eigenständige Dimensionen und Facetten zu betrachten und diese im Rahmen weiterführender Analysen auf differentielle Effekte hin zu untersuchen. In einer weiteren Untersuchung konnten Pietsch und Tulowitzki (2017) differentielle Effekte einzelner Führungsfacetten auf die Unterrichtsgestaltung von Lehrkräften nachweisen und zeigten, dass diese vor allem mit der instruktionalen Führung positiv zusammenhängen.

\subsection{Lernzentriertes Leitungshandeln als Mehrebenenkonstrukt}

Führung bzw. Leadership ist ein Mehrebenenphänomen per se, das sich auf verschiedenen Ebenen des Systems Schule manifestieren kann (Bliese et al. 2002). Sie kann sich entfalten zwischen einer Schulleitung und dem Lehrerkollegium (Ebene: Kollektiv), der Schulleitung und Gruppen von Lehrkräften (Ebene: Gruppe), der Schulleitung und einzelnen Lehrkräften (Ebene: Dyade), aber auch auf allen drei Ebenen. Entsprechend ist eine Führungstheorie, die einzelne oder mehrere Analyseebenen vernachlässigt, unvollständig, sind Daten zur Führung, die unterschiedliche Ebenen nicht berücksichtigen, unzureichend, und sind Führungspraktiken, die einzelne Organisationsebenen nicht mit einbeziehen, ineffektiv (Yammarino und Dionne 2018).

Mit Blick auf empirische Forschungsarbeiten liegt eine Mehrebenenstruktur formal dann vor, wenn Informationen bzw. Daten einer Analyseebene in einer anderen geschachtelt sind, z.B. Personen in Gruppen oder Schulen in Regionen. Bereits vor rund 30 Jahren konnten Rowan et al. (1991) zeigen, dass bei der Befragung von Lehrkräften zur Führung durch Schulleitungen nur etwa 25\% der Variation zwischen Schulen, aber 75\% innerhalb von Schulen, zwischen Lehrkräften, liegt. Werden diese Ebenen, die in Schulen in der Regel gekoppelt sind, in Forschungsdesigns und Analysen nicht berücksichtigt, kann es einerseits zu einer Vielzahl methodischer Probleme (z. B. inkorrekte Signifikanzbefunde) kommen, da die einzelnen Beobachtungen nicht unabhängig voneinander sind (Hox et al. 2017). Andererseits kann je nach Fragestellung eine andere Analyseebene relevant sein (Yammarino und Dansereau 2008). Werden beispielsweise Lehrkräfte zum Leitungshandeln der Schulleitung befragt, stellt sich die Frage, ob das Leitungshandeln als Klima oder Kontext verstanden wird und ob der Fokus auf der Person der Lehrkraft oder derjenigen der Schulleitung liegt (Lüdtke et al. 2011; Marsh et al. 2012). Bezieht sich die Befragung der Lehrkräfte z.B. konkret auf die Schulleitung als Referenten (Beispielitem: „Die Schulleitung hospitiert regelmäßig im Unterricht."), handelt es sich um ein Klimakonstrukt, das nicht (ausschließlich) auf der Individualebene modelliert und berichtet werden sollte, da hier die geteilte Wahrnehmung der Lehrkräfte an einer Schule interessiert (Bureau et al. 2017).

Obwohl entsprechende methodische Ansätze und Verfahren mittlerweile sowohl zum State-of-the-Art der Organisations- als auch der empirischen Bildungsforschung gehören, sind mehrebenenanalytische Studien im Bereich der Führungsforschung 
bislang vergleichsweise selten zu finden (Batistic et al. 2017). So haben Dionne et al. (2014) festgestellt, dass zwischen 1990 und 2013 in weniger als jeder dritten Publikation zum Thema Führung die untersuchte Analyseebene genannt wurde und nur in etwa jeder fünften Publikation gängige Verfahren der Mehrebenenanalyse korrekt eingesetzt wurden. Für den Bereich der Führung an Schulen sowie, konkreter, des lernzentrierten Leitungshandelns, liegen Reviews, die sich ausschließlich mit mehrebenenanalytischen Fragen beschäftigen, bislang nicht vor. Gleichwohl berichten Boyce und Bowers (2018b) in einem Review zu Analysen, die auf den Datensätzen des US-amerikanischen Schools and Staffing Surveys beruhen, dass die überwiegende Anzahl (35 von 59) der vorliegenden Studien zum Thema Leadership for Learning keine bzw. nur wenig belastbare Schlüsse zulässt, da in diesen bei der Datenanalyse die Mehrebenenstruktur nicht angemessen berücksichtig wurde (Lack of multilevel modeling).

Gleichwohl wurden in den vergangenen Jahren einige Studien zum lernzentrierten Leitungshandeln veröffentlicht, in denen elaborierte methodische Ansätze zum Umgang mit geschachtelten Datenstrukturen zum Einsatz kamen und die aufzeigen, welche Möglichkeiten mit Blick auf die diesbezügliche (quantitative) Datenanalyse bestehen (für eine Übersicht möglicher und im Folgenden aufgeführter Ansätze siehe Hox et al. 2017). So liegen mittlerweile vermehrt Untersuchungen vor, in deren Rahmen Hierarchisch Lineare Modelle genutzt werden (z. B. Witziers et al. 2003; Ma und Marion 2019). Weiterhin kommen zum Teil auch (konfirmatorische) Mehrebenen-Faktoranalysen zum Einsatz, um Unterschiede in der faktoriellen Struktur der einzelnen Dimensionen und Facetten lernzentrierten Leitungshandelns auf unterschiedlichen Analysebenen zu evaluieren (Dyer et al. 2005). So haben beispielsweise Boyce und Bowers (2018a) mithilfe dieses Ansatzes und anhand von Daten des 2011/2012er Schools and Staffing Surveys festgestellt, dass sich die Faktorstrukturen des Konstruktes auf Ebene einzelner Lehrkräfte und der Schulebene deutlich voneinander unterscheiden. Während auf der Individualebene sechs Faktoren nachgewiesen werden konnten, fanden sich auf der Schulebene nur drei Faktoren. Auffällig war dabei, dass der Faktor ,Instruktionale Führung ' nur auf Ebene der Schule bzw. des Kollektivs nachgewiesen werden konnte, woraus Boyce und Bowers (2018a, S. 16) schließen: „This supports the conceptualization of educational leadership as an organizational function rather than a specific collection of tasks and activities."

Erst seit kurzem finden sich darüber hinaus Mehrebenen-Latent-Class-Analysen, mit deren Hilfe Typologien lernzentrierten Leitungshandelns, unter Berücksichtigung der Mehrbenenstrukutur der Daten, voneinander unterschieden werden können (Vermunt 2003). Grundlegend ist hier vor allem die Annahme, dass Antworten von Lehrkräften zum Führungshandeln an der Schule durch den Kontext bzw. das jeweilige, individuelle Schulsetting bedingt sind (Zumbo et al. 2015). In diesem Sinne haben Bowers et al. (2017) Daten des Comprehensive Assessment of Leadership for Learning (CALL) genutzt und festgestellt, dass sich die Einschätzungen von Lehrkräften zum lernzentrierten Leitungshandeln an Schulen auf der Individualebene in drei Gruppen unterteilen lassen, wobei sich diese Gruppen jedoch unterschiedlich auf einzelne Schulen verteilen, also Unterschiede mit Blick auf einzelne Gruppen von Lehrkräften innerhalb von Schulen zu beobachten sind. Dabei weisen die Befunde darauf hin, dass es insbesondere mit Blick auf Schulentwicklungs- und 
Fortbildungsmaßnahmen unterschiedlicher Ansätze bedarf, die auf die Bedarfe der jeweiligen Schulen und der innerschulischen Akteure abgestimmt sind.

Selten zu finden sind bislang auch Mehrebenen-Strukturgleichungsmodelle, die, wie auch Hierarchisch Lineare Modelle, die verschiedenen Ebenen der Führung an Schulen berücksichtigen, darüber hinaus aber auch latente Variablen bzw. Faktoren modellieren zu können (Preacher et al. 2010). Neuere Ansätze ermöglichen es hierbei zu berücksichtigen, dass sich lernzentriertes Leitungshandeln in eine kollektiv-strukturelle und eine individuell-psychologische Komponente trennen lässt. Diese doppelt-latenten (doubly-latent) Modelle, modellieren Mess- und Strukturmodell auf der Individual- sowie der Gruppen- und/oder der Kollektivebene und ermöglichen es, mit Messfehlern und Strichprobenproblemen umzugehen (Marsh et al. 2012). Darüber hinaus ermöglicht es dieser Ansatz insbesondere mit Blick auf Klimakonstrukte zu evaluieren, in wie weit diese auf geteilten Wahrnehmungen von Lehrkräften in Schulen beruhen und in welchem Maße individuelle Wahrnehmungen von derjenigen des Gesamtkollegiums abweichen - ein Hinweis auf weitere Subklimas zum lernzentrierten Handeln in (Sub-)Gruppen innerhalb des Kollektivs (z. B. Fachgruppen im Gesamtkollegium) an Schulen (Bureau et al. 2017).

Eine solche Untersuchung mit Blick auf lernzentriertes Leitungshandeln haben Pietsch et al. (2019b) durchgeführt und anhand einer Stichprobe von 126 Schulen aus Deutschland gezeigt, dass es substanzielle Unterschiede im Zusammenhang einzelner Führungsdimensionen und der Arbeitszufriedenheit und dem Organisationalen Commitment von Lehrkräften auf der Individual und auf der Schulebene gibt. Während diese Variablen auf beiden untersuchten Ebenen stark positiv mit der verteilten Führung zusammen hingen, konnten Zusammenhänge mit transformationaler Führung nur auf der Individualebene festgestellt werden. Für die Dimension der instruktionalen Führung fanden sich hingen auf beiden Ebenen keine statistisch signifikanten und/oder praktisch relevanten Effekte. Pietsch und Leist (2019) zeigten darüber hinaus, dass alle Dimensionen lernzentrierten Leitungshandelns auch durch den regionalen Kontext beeinflusst werden: Je größer der Wettbewerb um Schüler*innen im Einzugsgebiet einer Schule, desto ausgeprägter das instruktionale und transformationale Leitungshandeln der Schulleitungen sowie die Beteiligung von Lehrkräften an Steuerungsentscheidungen.

\subsection{Mediationsbeziehung zwischen lernzentriertem Leitungshandeln und Outcome}

Mittlerweile lautet eine Grundannahme im Bereich der internationalen Schulleitungsforschung, dass Effekte auf Schüler*innenleistungen bzw. das Lernen an Schulen vor allem vermittelt zu erwarten sind (Scheerens 2012). Entsprechend ist die Wahl des richtigen bzw. zur Fragestellung passenden Wirkmodells von großer Bedeutung. Neben klassischen Modellen, die direkte Beziehungen im Sinne eines Black-Box-Ansatzes (Scriven 1994), in den Blick nehmen, finden sich Modelle, die ausschließlich indirekte, sowohl direkte als auch indirekte, aber auch solche, die darüber hinaus noch Kontextmerkmale und/oder Voraussetzungen des Leitungshandelns berücksichtigen (De Maeyer et al. 2007). Letztere Modelle haben entsprechend den Anspruch, nicht nur festzustellen, ob ein Zusammenhang von Leitungshandeln 
mit abhängigen Faktoren vorliegt, sondern versuchen Erklärungen für diese Zusammenhänge zu finden bzw. konfirmatorisch zu belegen (VanderWeele 2015). Variablen können in diesen Modellen komplexe Beziehungen haben, z. B. gleichzeitig abhängige und unabhängige Variable sein. Es wird dann z. B. davon ausgegangen, dass das Leitungshandeln seine Wirkung auf eine interessierende abhängige (distale) Variable, wie die Schüler*innenleistung, über eine vermittelnde (proximale) Variable, wie die Kooperation im Lehrerkollegium, entfaltet. Eine moderierte Beziehung liegt dabei dann vor, wenn die vermittelnde Variable Stärke und/oder Richtung des Zusammenhangs der Variablen beeinflusst (Baron und Kenny 1986), dies ggf. auch über verschiedene Ebenen der Organisation Schule hinweg (Preacher et al. 2010).

Strukturgleichungsmodelle gehören entsprechend mittlerweile zum Standardrepertoire in der empirischen Erforschung des Leitungshandelns an Schulen und entsprechende Analysen haben gezeigt, dass sich die von Leithwood et al. (2002) theoretisch postulierten Vermittlungsdimensionen auch empirisch nachweisen lassen (zur Übersicht Scheerens 2012). Für andere (in der Regel heuristische) Modelle, wie z. B. das aktuell diskutierte Four-Paths-Modell (Leithwood et al. 2017) liegt bislang hingegen keine ausreichende empirische Validierung aus vergleichenden Studien vor (Leithwood et al. 2019a, 2019b). Gleichwohl wurden die zugrunde liegenden Annahmen zumeist nicht im Rahmen des Leadership for Learning-Ansatzes überprüft, sondern beruhen primär auf theoretischen Überlegungen zur Veränderung (Change) von Schule, die daher zumeist wiederum im Rahmen von Studien zur transformationalen Führung an Schulen validiert wurden (z. B. Leithwood und Jantzi 2006; Thoonen et al. 2011). Unklar ist daher, ob alle Führungsfacetten gleichermaßen indirekt wirken oder ob es Unterschiede in den Wirkzusammenhängen zwischen einzelnen Führungsaspekten, Mediatoren und Effektvariablen gibt.

Bislang liegt keine Studie vor, die dieser Frage mit Blick auf Schüler*innenleistungen nachgeht und untersucht, ob, und falls ja, welche, Beziehungen zwischen einzelnen Führungsaspekten, Mediatorvariablen und Schüler*innenleistungen vorliegen. Einzig Boberg und Bourgeois (2016) weisen, anhand einer anfallenden Stichprobe von Schulen (convenient sample) nach, dass Leadership for Learning einen über die kollektiven Selbstwirksamkeitserwartungen (als relevanter Teilaspekt der innerschulischen Innovations- bzw. Entwicklungskapazität) von Lehrkräften vermittelten Effekt auf Leistungen von Schüler*innen hat. Dabei nutzen sie zur Messung des Leitungshandelns die von Leithwood und Jantzi (2006) entwickelte Transformational School Leadership Scale (TSL), die neben Aspekten transformationaler auch solche geteilter Führung erfasst, und erheben darüber hinaus Aspekte instruktionaler Führung. Differentielle Effekte bzw. Mediationen werden jedoch nicht berichtet, da aus den einzelnen Facetten des Führungshandelns ein Gesamtfaktor Integrated Transformational Leadership erstellt wurde. Hallinger und Heck (2010b) geben in einer weiteren Studie an, den Zusammenhang von lernzentriertem Leitungshandeln, innerschulischer Entwicklungskapazität und Schüler*innenleistungen zu untersuchen. Jedoch nutzen Sie im Rahmen der Studie nur eine Skala zur Messung des Leitungshandelns, die sich auf den Aspekt der geteilten Führung bezieht und die ,fails to measure certain potentially important facets of instructional leadership“ (Hallinger und Heck 2010b, S. 663). Die Befunde zeigen daher (nur), dass geteilte Führung einen positiven Einfluss auf die Lernzuwächse von Schüler*innen hat, der 
über die Entwicklungskapazität des Lehrerkollegiums vermittelt wird. Paletta et al. (2017) wiederum nutzen Skalen, die sowohl Aspekte transformationaler als auch instruktionaler Führung erfassen, erheben jedoch keine Informationen zur geteilten Führung an Schulen. Im Rahmen ihrer Studie untersuchen die Autoren darüber hinaus nur den Einfluss des Leitungshandelns auf ausgewählte Mediatorvariablen Schulklima sowie Selbstwirksamkeitsüberzeugungen und Arbeitszufriedenheit von Lehrkräften und weisen entsprechende Zusammenhänge nach. In wie weit diese potenziellen Mediatoren wiederum mit Schüler*innenleistungen zusammenhängen, wird hingegen nicht untersucht. Da hier zur Messung des lernzentrierten Leitungshandelns ein Faktor zweiter Ordnung generiert wurde, lassen sich darüber hinaus keine differentiellen Zusammenhänge feststellen.

Auch für Deutschland liegen keine belastbaren Befunde zum Thema vor. Einzig Pietsch et al. (2016) zeigen unter Zuhilfenahme eines Propensity Score Matchings und anhand von Daten des Hamburger Bildungsmonitorings, dass an Schulen, an denen über einen längeren Zeitraum und über verschiedene Schüler*innenkohorten hinweg hohe Lernzuwächse zu beobachten sind, eine besonders ausgeprägte instruktionale Führung nachzuweisen ist, sich andere Führungspraktiken zwischen diesen Schulen, und solchen mit geringen Lernzuwächsen, hingegen nicht unterscheiden. Ebenso wie Pietsch und Tulowitzki (2017) zeigen die Autoren darüber hinaus, dass der Zusammenhang zwischen einzelnen Führungsaspekten und der Unterrichtsgestaltung von Lehrkräften, die als vermittelnde Variable für Schüler*innenleistungen betrachtet wird, nur mit Blick auf die transformationale sowie die geteilte Führung indirekter Art ist; Zusammenhänge mit dem Teilaspekt der instruktionalen Führung hingegen waren in beiden Untersuchungen direkter Art.

\subsection{Lernzentriertes Leitungshandeln als Intervention, die zu Veränderungen führt}

Lernzentriertes Leitungshandeln ist ergebnisorientiert ausgerichtet und soll Entwicklungen und Veränderungen auf allen Ebenen des Systems Schule ermöglichen, indem das Lernen in den Mittelpunkt des Leitungshandelns gerückt wird (Daniëls et al. 2019). Dabei wird Leadership for Learning als Intervention betrachtet, die zu Entwicklungen und Veränderungen auf all diesen Ebenen führt und im Laufe der Zeit in verbesserten Schüler*innenleistungen mündet (Hallinger 2011; Murphy et al. 2007). Da wirkungsorientiertes Leitungshandeln auf sozialer Interaktion basiert, sind Effektannahmen darüber hinaus auch nie ausschließlich unidirektional sondern reziprok zu denken (Ogawa und Bossert 1995). Insofern unterstellt das Konzept eine (rekursive) Kausalität von Leitungshandeln und weiteren (vermittelnden) innerschulischen Merkmalen sowie Schüler*innenleistungen und es wird angenommen, dass Unterschiede und Veränderungen im Leitungshandeln mit Lern- und Entwicklungsunterschieden auf Ebene der Organisation Schule, derjenigen des Lehrerkollegiums sowie auf der von Schüler*innen einhergehen (Hallinger und Heck 2011a).

Beim lernzentrierten Leitungshandeln handelt es sich folglich um eine komplexe und komplizierte Intervention (Rogers 2008), deren Erforschung hohe Ansprüche an das forschungsmethodische Vorgehen stellt, da angenommen wird, dass a) lernzentriertes Leitungshandeln kausale Effekte nach sich zieht, b) sich diese Effek- 
te im Laufe der Zeit manifestieren und c) sich die einzelnen Modellvariablen im Veränderungsprozess ggf. wechselseitig beeinflussen. Berücksichtigt werden muss in Forschungsarbeiten daher insbesondere die zeitliche und kausale Ordnung, die Ursache-Wirkungsbeziehung(en) (Hallinger und Heck 2011a), die kontrafaktische Frage „was-wäre-wenn“ (z. B. ,,... das Leitungshandeln an der Schule anders gewesen wäre“, Holland 1986; Morgan und Winship 2015) sowie der Zeithorizont in der Effekte überhaupt zu erwarten sind bzw. sich manifestieren können (Ogawa und Bossert 1995; Shamir 2011; Goff und Finch 2016). Neben längsschnittlich angelegten Experimentalstudien sowie entsprechenden Quasi-Experimenten können dabei Verfahren zum Einsatz kommen, die darauf ausgelegt sind entsprechende Designs im Nachhinein (post-hoc) empirisch bzw. statistisch nachzuahmen (Avolio et al. 2009; Hiller et al. 2011). Querschnittstudien hingegen sind per se nur bedingt dazu geeignet die intendierten Fragestellungen zu beantworten, da diese insbesondere die Zeitkomponente vernachlässigen (Castillo und Trinh 2018). Darüber hinaus bieten vor allem Mixed-Method-Ansätze bei komplexen Fragestellungen zu Effekten von Leitungshandeln bzw. Wirkbeziehungen, das Potenzial kausalanalytische Fragestellungen zu beantworten (Teddlie 2005; Stentz et al. 2012).

Grundsätzlich zeigt eine Vielzahl von Studien den Zusammenhang von Leitungshandeln an Schulen und Schüler*innenleistungen sowie vermittelnden Variablen auf Ebene von Schule und Lehrerschaft. So haben beispielsweise Robinson et al. (2008) im Rahmen einer Metaanalyse 27 Studien zusammengetragen, die diesen Zusammenhang empirisch untersuchen. Gleichwohl stammen die wenigsten Befunde zum Thema aus Studien, in denen experimentelle oder quasi-experimentelle Längsschnitts- und/oder Messwiederholungsdesigns genutzt wurden. So berichten Goff und Finch (2016), dass derzeit nur zwei Studien (Heck und Moriyama 2010; Corcoran et al. 2012) vorliegen, die einem entsprechenden methodischen Anspruch gerecht werden und dabei auch Leistungsdaten von Schüler*innen nutzen. In einer dieser Studien gehen Heck und Moriyama (2010) dann auch explizit von einem lernzentrierten Leitungshandeln aus und untersuchen mithilfe eines Regression Discontinuity-Ansatzes (Imbens und Lemieux 2008) den Zusammenhang von Leitungshandeln, der Unterrichtsgestaltung durch Lehrkräfte und den Lernzuwächsen von Schüler*innen von der vierten zur fünften Klassenstufe. Im Rahmen der Studie zeigen Sie, dass lernzentriertes Leitungshandeln einen Einfluss auf die Unterrichtsgestaltung von Lehrkräften hat, die wiederum zu einer Steigerung von Schüler*innenleistungen führt.

Auch reziproke Effektmodelle gehören derzeit nicht zum Standard der internationalen empirischen Schulleitungsforschung. So berichtet Muijs (Muijs 2011), dass nur ca. $15 \%$ aller bislang veröffentlichten Studien zum Thema entsprechende Modelle nutzen. Eine der wenigen Ausnahmen hierzu haben Hallinger und Heck (2011a) vorgelegt. Im Rahmen einer Studie vier Messzeitpunkten untersuchen Sie mithilfe autoregressiver Modelle den reziproken Zusammenhang von Führung an Schulen, der Innovationskapazität von Lehrkräften und dem Lernzuwachs von Schüler*innen und zeigen, dass sich das Leitungshandeln an Schulen im Laufe der Zeit verändern kann und sich Führung und Innovationskapazität im Laufe der Zeit wechselseitig beeinflussen. Auch das Thema Zeit spielt außerhalb der genannten Studien bislang kaum eine Rolle bei der Erforschung lernzentrierten Leitungshandelns, wobei 
dies ein generelles Problem im Bereich der Führungsforschung ist (Shamir 2011; Day 2014; Castillo und Trinh 2018). So ist bekannt, dass verschiedene Ziele unterschiedlich lange benötigen, bis sie erreicht werden und dass dynamische Prozesse eine zeitliche Abfolge voraussetzen (Shamir 2011; Fischer et al. 2017). Gleichwohl werden Zeithorizonte sowie die Nachhaltigkeit mutmaßlicher Effekte in der Regel nicht thematisiert.

Studien, die Mixed-Method-Ansätze in größerem Umfang nutzen, liegen bislang ebenfalls nur vereinzelt vor. In einer der wenigen Ausnahmen zeigen Day et al. (2016) anhand einer Stichprobe von 20 Schulen, die über einen Zeitraum von drei Jahren begleitet wurden, das Potenzial dieses Ansatzes für die Forschung zum lernzentrierten Leitungshandeln auf. In einer Mischung aus Kontextdaten, Fragebogenerhebungen, Interviews und Schulbesuchen wurden Phasen der Schulentwicklung herausgearbeitet und in Relation zu den Lernergebnissen von Schüler*innen gesetzt. Dabei konnten die Autoren nachweisen, dass sowohl instruktionale als auch transformationale Führungspraktiken relevant für die Steigerung von Schüler*innenleistungen sind, sich die Gewichtung dieser Praktiken aber in Abhängigkeit vom Kontext einer Schule unterscheidet, und dass lernzentriertes Leitungshandeln und andere innerschulische Faktoren in einer dynamischen, reziproken Beziehung zueinander stehen.

Mit Blick auf Deutschland konstatierte Bonsen noch vor knapp einem Jahrzehnt (2010, S. 93), dass in der Schulleitungsforschung keine Forschungsergebnisse vorlägen, ,die über die Ausleuchtung von Fragmenten der sozialen Realität von Schulleitungen hinauszugehen vermögen“. Zwar wurden in den vergangenen Jahren diverse qualitativ orientierte Studien (u. a. Bonsen 2003; Roggenbuck-Jagau 2005; Muslic et al. 2013; Bärstecher 2014; Ramsteck et al. 2015), Analysen größerer Datensätze (Klein 2015, 2016b) und quantitative bzw. mixed-methods Erhebungen mit größeren Fallzahlen (Warwas 2012; Huber et al. 2013; Brauckmann 2014) durchgeführt. Auch wurden verschiedene Aspekte rund um die Wirkung von Leitungshandeln untersucht (z. B. Leitungshandeln im Kontext von Lehrergesundheit (Gerick 2014), Zusammenhänge zwischen Leitungshandeln und Nutzung von Ergebnisrückmeldungen aus Vergleichsarbeiten (Kronsfoth et al. 2018) oder der Einfluss von Schulleitungshandeln auf Unterrichtsqualität (Bonsen et al. 2002)). Gleichwohl liegen aus Deutschland aktuell keine Studien vor, die den Zusammenhang von (lernzentriertem) Leitungshandeln und Schüler*innenleistungen im zeitlichen Verlauf untersuchen. So wurde im Rahmen der bislang einzig vorliegenden Untersuchung aus dem deutschsprachigen Raum in deren Rahmen Lernzuwächse von Schüler*innen in Beziehung zum Leitungshandeln gesetzt wurden (Pietsch et al. 2016), zwar zur post-hoc-Erzeugung eines quasi-experimentellen Designs ein Propensity Score Matching-Verfahren genutzt. Jedoch wurde dabei nicht die individuelle Lernentwicklung von Schüler*innen im Längsschnitt modelliert, sondern ausschließlich evaluiert, inwiefern sich das Leitungshandeln an hoch-performanten Schulen von demjenigen an nicht hoch-performanten Schulen unterscheidet. Die einzige uns bekannte Untersuchung aus Deutschland zum Thema (Pietsch et al. 2019a), die mehrere Messzeitpunkte umfasst, bezieht sich hingegen nicht auf Schüler*innenleistungen, sondern untersucht den Zusammenhang von Leitungshandeln im Kontext von Schulleitungswechseln sowie deren (kombinierte) Effekte auf die Veränderung der Unterrichtsqualität an 
Grundschulen und zeigt, dass bei Leitungswechseln eine zu starke Fokussierung auf den Aspekt der unterrichtsbezogenen Führung mit einer negativen Entwicklung der Unterrichtsqualität einher gehen, wohingegen die Verteilung von Führungsverantwortung im Kollegium zu einer Stabilisierung beitragen kann.

\section{Schlussbetrachtung}

Lernzentriertes Leitungshandeln an Schulen prägt auf internationaler Ebene den fachwissenschaftlichen Diskurs zum Thema effektive Führung von Schulen. Im deutschsprachigen Raum wird das Konzept hingegen bislang kaum behandelt, weder theoretisch, noch empirisch. Eine zentrale Frage lautet somit: Lassen sich auch in den deutschsprachigen Ländern ähnliche Zusammenhänge schulischen Leitungshandelns mit Lernentwicklungen seitens der Schüler*innen erwarten und nachweisen, wie in anderen, insbesondere anglophonen Ländern?

Wie wir gezeigt haben, ist dabei nicht per se zu erwarten, dass diese Zusammenhänge unidirektionaler Art sind. Vielmehr muss davon ausgegangen werden, dass sich entsprechende Effekte im Laufe der Zeit in der sozialen Interaktion der Schulbeteiligten ausbilden und somit in reziproken Beziehungen zueinanderstehen. Leitungshandeln, das Schüler*innen erreicht, wird darüber hinaus über eine Vielzahl von innerschulischen Variablen, die sowohl Stärke als auch Richtung der Wirkung beeinflussen können, vermittelt. Diesbezüglich hat sich in internationalen Studien, aber auch den wenigen Untersuchungen aus dem deutschsprachigen Raum, insbesondere die Innovationskapazität der Organisation Schule sowie diejenige von Lehrkräften als wichtigster vermittelnder Faktor erwiesen (Leithwood und Jantzi 2006; Hallinger und Heck 2010a; Pietsch et al. 2016; vgl. hierzu auch die Ausführungen zur Schulentwicklungskapazität, z.B. Maag Merki 2017): Die Fähigkeit und die Erwartungen des Lehrerkollegiums Entwicklungen in Gang sowie Veränderungen und Innovationen in Schule und Unterricht umsetzen zu können, entscheiden demnach maßgeblich mit, ob sich lernzentriertes Leitungshandeln im Unterricht von Lehrkräften und darüber vermittelt im Lernen von Schüler*innen niederschlägt oder nicht. Maßnahmen, die auf die Stärkung der kollektiven Wirksamkeit im Kollegium, also zur Entwicklung der Förderung der Kompetenzen, der Erhöhung der Motivation sowie der ihnen zur Verfügung stehenden Ressourcen beitragen, sind somit im Hinblick auf die Verbesserung von Leistungen von Schüler*innen (Fullan 2006) ebenso relevant wie die Ausrichtung schulischer Führung auf das Lernen (Hallinger und Heck 2011b).

Eine große Herausforderung für empirische Studien zum Thema stellt die Komplexität der theoretischen (Modell-)Annahmen dar. Selbst im internationalen Raum ist es bislang nicht gelungen, dem Leadership for Learning-Konzept umfassend gerecht zu werden, da dessen empirische Erforschung äußerst voraussetzungsreich ist. Grundlegende Voraussetzung, um Lernen bzw. Entwicklungen und Veränderungen verlässlich zu messen, ist dabei die Erhebung von Daten in Längsschnitt- oder zumindest Messwiederholungsdesigns. Darüber hinaus müssen einerseits Daten auf verschiedenen Ebenen des Systems Schule erhoben sowie reziproke Wirkbeziehungen auf und ggf. zwischen den verschiedenen Ebenen aber auch die Mehrdimen- 
sionalität des Führungskonstrukts berücksichtigt werden. Hierbei ist insbesondere zu berücksichtigen, dass es in Schulen zu ständigen Fluktuationen kommt: Schüler*innen, Lehrkräfte aber auch Schulleitungen selbst können zwischen Schulen wechseln oder das System ganz verlassen. Wie sich diese Prozesse an Schulen in Deutschland gestalten ist bislang kaum erforscht, insbesondere da es keine Datensätze gibt, die es ermöglichen die Bildungs- und Karriereverläufe von Lehrkräften und Schulleitungen nachzuverfolgen.

Mit Blick auf die zukünftige Erforschung lernzentrierten Leitungshandelns an Schulen im deutschsprachigen Raum empfehlen wir daher folgende Agenda:

1. Daten zur Führung an Schulen müssen als mehrdimensionales Konstrukt erfasst werden. Dafür muss das Führungskonzept des lernzentrierten Leitungshandelns für den deutschsprachigen Raum messbar gemacht und ggf. mit Blick auf die landesspezifischen Anforderungen angepasst werden (eine Vorlage kann ggf. das Comprehensive Assessment of Leadership for Learning (CALL), Blitz et al. 2014, sein).

2. Daten zur Führung an Schulen müssen immer durch Informationen zu schulischen und führungsrelevanten Kontexten ergänzt werden. Diese können, je nach Fragestellung, den institutionellen, den unmittelbaren räumlichen, den sozio-kulturellen, den politischen, sowie den ökonomischen Kontext einer Schule sowie ggf. den Schulentwicklungskontext, der auch als einzelschulhistorischer Kontext bezeichnet werden kann, umfassen.

3. Daten zur Führung an Schulen müssen, wenn es um die Prüfung kausaler Zusammenhänge geht, im Rahmen von Experimentaldesigns bzw. Quasiexperimentaldesigns erhoben werden. Ist dies nicht möglich, müssen entsprechende Designs im Nachhinein durch angemessene statistische post-hoc-Verfahren nachgeahmt werden.

4. Daten zur Führung an Schulen müssen im Zusammenhang mit Daten zur Lernentwicklung von Schüler*innen erhoben werden. Idealerweise müssen darüber hinaus Daten zur Entwicklung innerschulischer Kapazitäten (insb. von Lehrkräften) wiederholt oder längsschnittlich erhoben werden. Mögliche Fluktuationen sind dabei zu berücksichtigen.

5. Daten zu vermittelnden Faktoren müssen auf verschiedenen Ebenen gemessen und modelliert werden; es müssen, wo sinnvoll, mögliche Interaktionen zwischen den verschieden Organisationsebenen (Dyade, Gruppe, Kollektiv) berücksichtigt werden.

Eine entsprechende Anbindung kann beispielsweise im Nationalen Bildungspanel (National Educational Panel Study - NEPS) aber auch im Bildungsmonitoring der Länder angelegt sein. Denkbar sind darüber hinaus Einzelstudien, die einen entsprechenden Fokus aufweisen. Hierbei ist jedoch wichtig, bereits vorab den Zeithorizont zu berücksichtigen und zu klären, welche Effekte in welchem Zeitraum zu erwarten sind und wie nachhaltig diese mutmaßlichen Effekte sein können (vgl. Shamir 2011; Fischer et al. 2017). Insbesondere Studien, die Mixed-Method-Ansätze nutzen und Schulen über einen längeren Zeitraum begleiten bieten darüber hinaus das Potenzial das Forschungsfeld voran zu bringen und Erklärungen für Zusammenhänge und Wirkungen zu liefern (Stentz et al. 2012; Day et al. 2016), da sie sowohl 
theorie-generierend als auch -überprüfend sein können. Aber auch theoriebasierte Einzelstudien zu einzelnen Teilaspekten können zur systematischen Erforschung lernzentrierten Leitungshandelns beitragen, sofern diese z. B. dem Ansatz der General-Elemination-Methode (GEM; Scriven 2008, 2009) folgen, bei dem es darum geht die bestmögliche Erklärung (,,inference to the best explanation“) für eine beobachtete Veränderung aufseiten einer abhängigen Variablen zu finden (Cook et al. 2009) bzw. alternative Erklärungen systematisch auszuschließen (,,rule out altenative explantions", Reichhardt 2011). Hierfür müssen in einem ersten Schritt mögliche Gründe für eine Veränderung der abhängigen Variablen spezifiziert werden, in einem zweiten Schritt die möglichen Modi Operandi (Wirkmechanismen) heraus gearbeitet werden, die beschreiben, wie diese möglichen Ursachen zu potenziellen Effekten führen, um abschließend, in einem dritten Schritt, das Vorhandensein der einzelnen Modi Operandi systematisch zu prüfen und alternative Erklärungen auszuschließen (Reichardt 2000). Ein besonderer Vorteil dieses Ansatzes besteht dabei darin, dass er keine kontrafaktischen Forschungsdesigns voraussetzt und es daher auch ermöglicht Befunde aus qualitativen und quantitativen Studien integrativ zu nutzen (Reichardt 2011).

Auf Ebene der Praxis wiederum erscheint es angesichts der im internationalen Kontext festgestellten Wirksamkeit aus unserer Sicht zentral, die Position der Schulleitung zu würdigen und zu stärken und den Aspekt der geteilten Führung stärker in den Blick zu nehmen. Dies umfasst einerseits die Klärung des Berufsbildes von Schulleitungen in Deutschland, eine aufgewertete verpflichtende Qualifizierungsphase (Tulowitzki et al. 2019), den Aufbau zielgerichteter Fortbildungsund Unterstützungsangebote sowie eine Klärung der Rolle der Schulaufsicht (Tulowitzki 2019). Andererseits können die Spielräume für die Übernahme geteilter Führungsverantwortung erweitert werden, z.B. durch eine Stärkung entsprechender bestehender Strukturen wie der erweiterten Schulleitung. Auch der schulischen Steuergruppe als intermediäre Instanz zwischen Organisationsebene und Professionsebene kommt in diesem Zusammenhang eine wichtige Rolle zu (vgl. Feldhoff 2011). In Bezug auf das berufliche Selbstverständnis erscheint es hilfreich, die (dynamischen) Führungsrollen sowie das Führungsverständnis von Lehrkräften (Printy und Marks 2006) zu klären und diese zur interdependenten Verantwortungsübernahme in Schulen zu befähigen (Houghton und Yoho 2005). Derartige Rahmenbedingen könnten dazu beitragen, auch im hiesigen Kontext die Wirksamkeit und Verbreitung lernzentrierten Leitungshandelns maßgeblich zu erhöhen.

Funding Open Access funding provided by Projekt DEAL.

Open Access Dieser Artikel wird unter der Creative Commons Namensnennung 4.0 International Lizenz veröffentlicht, welche die Nutzung, Vervielfältigung, Bearbeitung, Verbreitung und Wiedergabe in jeglichem Medium und Format erlaubt, sofern Sie den/die ursprünglichen Autor(en) und die Quelle ordnungsgemäß nennen, einen Link zur Creative Commons Lizenz beifügen und angeben, ob Änderungen vorgenommen wurden.

Die in diesem Artikel enthaltenen Bilder und sonstiges Drittmaterial unterliegen ebenfalls der genannten Creative Commons Lizenz, sofern sich aus der Abbildungslegende nichts anderes ergibt. Sofern das betreffende Material nicht unter der genannten Creative Commons Lizenz steht und die betreffende Handlung nicht nach gesetzlichen Vorschriften erlaubt ist, ist für die oben aufgeführten Weiterverwendungen des Materials die Einwilligung des jeweiligen Rechteinhabers einzuholen. 
Weitere Details zur Lizenz entnehmen Sie bitte der Lizenzinformation auf http://creativecommons.org/ licenses/by/4.0/deed.de.

\section{Literatur}

Avolio, B. J. (2003). Examining the full range model of leadership: looking back to transfer forward. In D. Day \& S. J. Zaccaro (Hrsg.), Leader development for transforming organizations: growing leaders for tomorrow (S. 71-98). Mahwah: Erlbaum.

Avolio, B. J., \& Bass, B. M. (1995). Individual consideration viewed at multiple levels of analysis: a multilevel framework for examining the diffusion of transformational leadership. The Leadership Quarterly, 6(2), 199-218.

Avolio, B. J., Reichard, R. J., Hannah, S. T., Walumbwa, F. O., \& Chan, A. (2009). A meta-analytic review of leadership impact research: experimental and quasi-experimental studies. The Leadership Quarterly, 20(5), 764-784. https://doi.org/10.1016/j.leaqua.2009.06.006.

Baron, R., \& Kenny, D. (1986). The moderator-mediator variable distinction in social psychological research: conceptual, strategic, and statistical considerations. Journal of Personality and Social Psychology, 51(6), 1173-1182. https://doi.org/10.1037//0022-3514.51.6.1173.

Bärstecher, F.E. (2014). Was machen Schulleiter tatsächlich und welche Faktoren beeinflussen diese ausgeführten Tätigkeiten?, What do school principals really do and which factors do influence these tasks? (University of Potsdam). https://publishup.uni-potsdam.de/frontdoor/index/index/docId/8010. Zugegriffen: 11. Aug. 2020.

Bass, B. M. (1990). From transactional to transformational leadership: learning to share the vision. Organizational Dynamics, 18(3), 19-31. https://doi.org/10.1016/0090-2616.

Bass, B. M., \& Avolio, B. J. (1993). Transformational leadership and organizational culture. Public Administration Quarterly, 17(1), 112-121.

Batistic, S., Cerne, M., \& Vogel, B. (2017). Just how multi-level is leadership research? A document cocitation analysis 1980-2013 on leadership constructs and outcomes. The Leadership Quarterly, 28(1), $86-103$.

Bliese, P.D., Halverson, R. R., \& Schriesheim, C. A. (2002). Benchmarking multilevel methods in leadership: the articles, the model, and the data set. Leadership Quarterly, 13(1), 3-14. https://doi.org/10. 1016/S1048-9843(01)00101-1.

Blitz, M. H., Salisbury, J., \& Kelley, C. (2014). The role of cognitive validity testing in the development of CALL, the comprehensive assessment of leadership for learning. Journal of Educational Administration, 52(3), 358-378. https://doi.org/10.1108/JEA-01-2013-0008.

Boberg, J.E., \& Bourgeois, S. J. (2016). The effects of integrated transformational leadership on achievement. Journal of Educational Administration, 54(3), 357-374. https://doi.org/10.1108/JEA-07-20140086.

Bonsen, M. (2003). Schule, Führung, Organisation. Eine empirische Studie zum Organisations- und Führungsverständnis von Schulleiterinnen und Schulleitern. Münster: Waxmann.

Bonsen, M. (2010). Schulleitungshandeln. In H. Altrichter \& K. Maag Merki (Hrsg.), Handbuch Neue Steuerung im Schulsystem (S. 277-294). Wiesbaden: VS.

Bonsen, M., von der Gathen, J., Iglhaut, C., \& Pfeiffer, H. (2002). Die Wirksamkeit von Schulleitung: Empirische Annäherungen an ein Gesamtmodell schulischen Leistungshandelns. Weinheim: Juventa.

Bowers, A. (2020). Examining a congruency-typology model of leadership for learning using two-level latent class analysis with TALIS 2008. OECD Education Working Paper No. 219. Paris: OECD.

Bowers, A. J., Blitz, M., Modeste, M.E., Salisbury, J., \& Halverson, R. (2017). Is there a typology of teacher and leader responders to CALL, and do they cluster in different types of schools? A two-level latent class analysis of CALL survey data. Teachers College Record, 119(4), 1-66.

Boyce, J., \& Bowers, A. J. (2018a). Different levels of leadership for learning: Investigating differences between teachers individually and collectively using multilevel factor analysis of the 2011-2012 Schools and Staffing Survey. International Journal of Leadership in Education, 21(2), 197-225. https://doi.org/10.1080/13603124.2016.1139187.

Boyce, J., \& Bowers, A. J. (2018b). Toward an evolving conceptualization of instructional leadership as leadership for learning: meta-narrative review of 109 quantitative studies across 25 years. Journal of Educational Administration, 56(2), 161-182. https://doi.org/10.1108/JEA-06-2016-0064.

Brauckmann, S. (2014). Ergebnisbericht im Rahmen des BMBF Forschungsschwerpunkts „Steuerung im Bildungssystem“ (SteBis) geförderten Forschungsprojekts „,Schulleitungshandeln zwischen erweiter- 
ten Rechten und Pflichten (SHaRP)“. Berlin: Deutsches Institut für Internationale Pädagogische Forschung.

Brauckmann, S., \& Schwarz, A. (2015). No time to manage? The trade-off between relevant tasks and actual priorities of school leaders in Germany. International Journal of Educational Management, 29(6), 749-765. https://doi.org/10.1108/IJEM-10-2014-0138.

Brauckmann, S., Geissler, G., Feldhoff, T., \& Pashiardis, P. (2016). Instructional leadership in Germany: an evolutionary perspective. International Studies in Educational Administration, 44(2), 5-20.

Bureau, J.S., Gagné, M., Morin, A. J.S., \& Mageau, G. A. (2017). Transformational leadership and incivility: a multilevel and longitudinal test. Journal of Interpersonal Violence. https://doi.org/10.1177/ 0886260517734219.

Burns, J. M. (1978). Leadership. New York: Harper \& Row.

Bush, T., \& Glover, D. (2014). School leadership models: what do we know? School Leadership \& Management, 34(5), 553-571. https://doi.org/10.1080/13632434.2014.928680.

Castillo, E. A., \& Trinh, M. P. (2018). In search of missing time: a review of the study of time in leadership research. The Leadership Quarterly, 29(1), 165-178. https://doi.org/10.1016/j.leaqua.2017.12.001.

Cook, T.D., Scriven, M., Coryn, C.L.S., \& Evergreen, S. D. H. (2009). Contemporary thinking about causation in evaluation: a dialogue with Tom cook and Michael Scriven. American Journal of Evaluation, 31(1), 105-117. https://doi.org/10.1177/1098214009354918.

Corcoran, S., Schwartz, A., \& Weinstein, M. (2012). Training your own: the impact of New York City's aspiring principals program on student achievement. Educational Evaluation and Policy Analysis, 34(2), 232-253. https://doi.org/10.3102/0162373712437206.

Daniëls, E., Hondeghem, A., \& Dochy, F. (2019). A review on leadership and leadership development in educational settings. Educational Research Review, 27, 110-125. https://doi.org/10.1016/j.edurev. 2019.02.003.

Day, D. V. (2014). Time and leadership. In A.J. Shipp \& Y. Fried (Hrsg.), How time impacts groups, organizations and methodological choices (Time and work, Bd. 2, S. 30-52). New York: Psychology Press.

Day, C., Gu, Q., \& Sammons, P. (2016). The impact of leadership on student outcomes how successful school leaders use transformational and instructional strategies to make a difference. Educational Administration Quarterly, 52(2), 221-258. https://doi.org/10.1177/0013161X15616863.

De Maeyer, S., Rymenans, R., Van Petegem, P., van den Bergh, H., \& Rijlaarsdam, G. (2007). Educational leadership and pupil achievement: The choice of a valid conceptual model to test effectsin school effectiveness research. School Effectiveness and School Improvement, 18(2), 125-145. https://doi. org/10.1080/09243450600853415.

Dionne, S. D., Gupta, A., Sotak, K. L., Shirreffs, K. A., Serban, A., Hao, C., \& Yammarino, F. J. (2014). A 25-year perspective on levels of analysis in leadership research. Leadership Quarterly, 25(1), 6-35. https://doi.org/10.1016/j.leaqua.2013.11.002.

Dyer, N. G., Hanges, P. J., \& Hall, R. J. (2005). Applying multilevel confirmatory factor analysis techniques to the study of leadership. The Leadership Quarterly, 16(1), 149-167. https://doi.org/10.1016/j. leaqua.2004.09.009.

Feldhoff, T. (2011). Schule organisieren. https://doi.org/10.1007/978-3-531-93384-9.

Feldhoff, T., Rolff, H.-G., \& Kanders, M. (2008). Schulleitung und innere Schulorganisation. In H. G. Holtappels, K. Klemm \& H.-G. Rolff (Hrsg.), Schulentwicklung durch Gestaltungsautonomie. Ergebnisse der Begleitforschung zum Modellvorhaben „Selbstständige Schule“ in Nordrhein-Westfalen (S. 146-173). Münster: Waxmann.

Felfe, J. (2006). Transformationale und charismatische Führung - Stand der Forschung und aktuelle Entwicklungen. Zeitschrift für Personalpsychologie, 5(4), 163-176. https://doi.org/10.1026/1617-6391. 5.4.163.

Fischer, T., Dietz, J., \& Antonakis, J. (2017). Leadership process models: a review and synthesis. Journal of Management, 43(6), 1726-1753. https://doi.org/10.1177/0149206316682830.

Fullan, M. (2006). The future of educational change: system thinkers in action. Journal of Educational Change, 7(3), 113-122. https://doi.org/10.1007/s10833-006-9003-9.

Gerick, J. (2014). Führung und Gesundheit in der Organisation Schule: Zur Wahrnehmung transformationaler Führung und die Bedeutung für die Lehrergesundheit als Schulqualitätsmerkmal. Münster: Waxmann.

Goff, P., \& Finch, M. (2016). Challenges and opportunities for education leadership scholarship: a methodological critique. In A. J. Bowers, A. R. Shoho \& B. G. Barnett (Hrsg.), Challenges and opportunities of educational leadership research and practice: the state of the field and its multiple futures (Bd. 6 , S. 119-146). Charlotte: Information Age. 
Goldring, E., Cravens, X., Porter, A., Murphy, J., \& Elliott, S. (2015). The convergent and divergent validity of the Vanderbilt Assessment of Leadership in Education (VAL-ED): Instructional leadership and emotional intelligence. Journal of Educational Administration, 53(2), 177-196. https://doi.org/10. 1108/JEA-06-2013-0067.

Hallinger, P. (1994). A resource manual for the principal instructional management rating scale (PIMRS manual 2.2). Nashville: Center for the Advanced Study of Educational Leadership.

Hallinger, P. (2003). Leading educational change: reflections on the practice of instructional and transformational leadership. Cambridge Journal of Education, 33(3), 329-352.

Hallinger, P. (2011). Leadership for learning: Lessons from 40 years of empirical research. Journal of Educational Administration, 49(2), 125-142. https://doi.org/10.1108/09578231111116699.

Hallinger, P. (2016). Bringing context out of the shadows of leadership. Educational Management Administration \& Leadership, 46(1), 5-24. https://doi.org/10.1177/1741143216670652.

Hallinger, P., \& Heck, R.H. (2010a). Collaborative leadership and school improvement: understanding the impact on school capacity and student learning. School Leadership \& Management, 30(2), 95-110. https://doi.org/10.1080/13632431003663214.

Hallinger, P., \& Heck, R. H. (2010b). Leadership for learning: does collaborative leadership make a difference in school improvement? Educational Management Administration \& Leadership, 38(6), 654-678. https://doi.org/10.1177/1741143210379060.

Hallinger, P., \& Heck, R. H. (2011a). Conceptual and methodological issues in studying school leadership effects as a reciprocal process. School Effectiveness and School Improvement, 22(2), 149-173.

Hallinger, P., \& Heck, R. H. (2011b). Exploring the journey of school improvement: classifying and analyzing patterns of change in school improvement processes and learning outcomes. School Effectiveness and School Improvement, 22(1), 1-27. https://doi.org/10.1080/09243453.2010.536322.

Hallinger, P., \& Wang, W.-C. (2015). Assessing instructional leadership with the principal instructional management rating scale. Cham: Springer.

Harazd, B. (2010). Schulleitungstypen in eigenverantwortlichen Schulen und ihr Zusammenhang zur Schulqualität. In N. Berkemeyer, W. Bos, H. G. Holtappels \& N. McElvany (Hrsg.), Jahrbuch der Schulentwicklung: Band 16. Daten, Beispiele und Perspektiven (S. 261-288). https://pure.mpg.de/ pubman/faces/ViewItemOverviewPage.jsp?itemId=item_2099685_5.

Harazd, B., \& van Ophuysen, S. (2011). Transformationale Führung in Schulen. Der Einsatz des „Multifactor Leadership Questionnaire“ (MLQ 5 x Short). Journal for educational research online, 3(1), $141-167$.

Harris, A. (2004). Distributed leadership and school improvement: leading or misleading? Educational Management Administration \& Leadership, 32(1), 11-24. https://doi.org/10.1177/1741143204039297.

Harris, A. (2008). Distributed leadership: according to the evidence. Journal of Educational Administration, 46(2), 172-188. https://doi.org/10.1108/09578230810863253.

Harris, A., \& Spillane, J.P. (2008). Distributed leadership through the looking glass. Management in Education, 22(1), 31-34.

Hartig, J., \& Höhler, J. (2009). Multidimensional IRT models for the assessment of competencies. Studies In Educational Evaluation, 35(2-3), 57-63. https://doi.org/10.1016/j.stueduc.2009.10.002.

Hattie, J. (2009). Visible learning: a synthesis of over 800 meta-analyses relating to achievement. New York: Routledge.

Hattie, J. (2015). High-impact leadership. Educational Leadership, 72(5), 36-40.

Heck, R.H., \& Moriyama, K. (2010). Examining relationships among elementary schools' contexts, leadership, instructional practices, and added-year outcomes: a regression discontinuity approach. School Effectiveness and School Improvement, 21(4), 377-408. https://doi.org/10.1080/09243453. 2010.500097.

Hersey, P., \& Blanchard, K.H. (1982). Management of organizational behavior (4. Aufl.). Upper Saddle River: Prentice-Hall.

Hiller, N. J., DeChurch, L. A., Murase, T., \& Doty, D. (2011). Searching for outcomes of leadership: a 25year review. Journal of Management, 37(4), 1137-1177. https://doi.org/10.1177/0149206310393520.

Holland, P.W. (1986). Statistics and causal inference. Journal of the American Statistical Association, 81(396), 945-960. https://doi.org/10.2307/2289064.

Houghton, J.D., \& Yoho, S. K. (2005). Toward a contingency model of leadership and psychological empowerment: when should self-leadership be encouraged? Journal of Leadership \& Organizational Studies, 11(4), 65-83. https://doi.org/10.1177/107179190501100406.

Hox, J., Moerbeek, M., \& Van de Schoot, R. (2017). Multilevel analysis: techniques and applications. New York: Routledge. 
Huber, S.G., Wolfgramm, C., \& Kilic, S. (2013). Vorlieben und Belastungen im Schulleitungshandeln: Ausgewählte Ergebnisse aus der Schulleitungsstudie 2011/2012 in Deutschland, Österreich, Liechtenstein und der Schweiz. In S. G. Huber (Hrsg.), Jahrbuch Schulleitung 2013 - Befunde und Impulse zu den Handlungsfeldern des Schulmanagements (S. 259-271). Köln: Carl Link.

Hulpia, H., Devos, G., \& Keer, H. V. (2009). The influence of distributed leadership on teachers' organizational commitment: a multilevel approach. The Journal of Educational Research, 103(1), 40-52. https://doi.org/10.1080/00220670903231201.

Imbens, G. W., \& Lemieux, T. (2008). Regression discontinuity designs: a guide to practice. Journal of Econometrics, 142(2), 615-635. https://doi.org/10.1016/j.jeconom.2007.05.001.

Kelley, R.C., Thornton, B., \& Daugherty, R. (2005). Relationships between measures of leadership and school climate. Education, 126(1), 17-25.

Klein, E. D. (2015). Zwillinge oder entfernte Verwandte? Strategien der Schulleitung in Schulen in deprivierter Lage in Deutschland und den USA. Zeitschrift für Bildungsforschung, 6(1), 5-23. https://doi. org/10.1007/s35834-015-0138-8.

Klein, E. D. (2016a). Instructional Leadership in den USA. Ein Modell für Schulleiterinnen und Schulleiter in Deutschland? Tertium Comparationis, 22(2), 203-229.

Klein, E.D. (2016b). Schulleitungshandeln an staatlichen Schulen in Deutschland und den USA: Eine vergleichende Analyse auf Basis von PISA-Daten. Zeitschrift für Erziehungswissenschaft, 20(01), 61-87. https://doi.org/10.1007/s11618-016-0695-1.

Kronsfoth, K., Muslic, B., Graf, T., \& Kuper, H. (2018). Der Zusammenhang zwischen Führungsdimensionen in der Schulleitung und der Nutzung von Ergebnisrückmeldungen aus Vergleichsarbeiten. DDS - Die Deutsche Schule, 110(1), 47-64. https://doi.org/10.31244/dds/2018.01.04.

Law, K.S., Wong, C.-S., \& Mobley, W.H. (1998). Toward a taxonomy of multidimensional constructs. Academy of Management Review, 23(4), 741-755.

Lee, M., Walker, A., \& Chui, Y.L. (2012). Contrasting effects of instructional leadership practices on student learning in a high accountability context. Journal of Educational Administration, 50(5), 586-611. https://doi.org/10.1108/09578231211249835.

Leithwood, K., \& Jantzi, D. (2005). A review of transformational school leadership research 1996-2005. Leadership and Policy in Schools, 4(3), 177-199. https://doi.org/10.1080/15700760500244769.

Leithwood, K., \& Jantzi, D. (2006). Transformational school leadership for large-scale reform: effects on students, teachers, and their classroom practices. School Effectiveness and School Improvement, 17(2), 201-227. https://doi.org/10.1080/09243450600565829.

Leithwood, K., \& Jantzi, D. (2009). A review of empirical evidence about school size effects: a policy perspective. Review of Educational Research, 79(1), 464-490. https://doi.org/10.3102/ 0034654308326158.

Leithwood, K., \& Louis, K. S. (2012). Linking leadership to student learning. San Francisco: Jossey-Bass.

Leithwood, K., \& Mascall, B. (2008). Collective leadership effects on student achievement. Educational Administration Quarterly, 44(4), 529-561. https://doi.org/10.1177/0013161X08321221.

Leithwood, K., Harris, A., \& Hopkins, D. (2020). Seven strong claims about successful school leadership revisited. School Leadership \& Management, 40(1), 5-22. https://doi.org/10.1080/13632434.2019. 1596077.

Leithwood, K., Jantzi, D., \& Mascall, B. (2002). A framework for research on large-scale reform. Journal of Educational Change, 3(1), 7-33. https://doi.org/10.1023/A:1016527421742.

Leithwood, K., Jantzi, D., \& Steinbach, R. (1999). Changing leadership for changing times. Philadelphia: Open University Press.

Leithwood, K., Sun, J., \& McCullough, C. (2019a). How school districts influence student achievement. Journal of Educational Administration, 57(5), 519-539. https://doi.org/10.1108/JEA-09-2018-0175.

Leithwood, K., Sun, J., \& Pollock, K. (Hrsg.). (2017). How school leaders contribute to student success. https://doi.org/10.1007/978-3-319-50980-8.

Leithwood, K., Sun, J., \& Schumacker, R. (2019b). How school leadership influences student learning: a test of "the four paths model". Educational Administration Quarterly. https://doi.org/10.1177/ $0013161 X 19878772$.

Leithwood, K., Louis, K. S., Anderson, S., \& Wahlstrom, K. (2004). Review of research: how leadership influences student learning. http://conservancy.umn.edu/handle/11299/2035. Zugegriffen: 29. Nov. 2019

Louis, K.S., Leithwood, K., Wahlstrom, K.L., \& Anderson, S. (2010a). Learning from leadership: investigating the links to improved student learning. https:/www.wallacefoundation.org/knowledgecenter/pages/investigating-the-links-to-improved-student-learning.aspx Commissioned by The Wallace Foundation. Zugegriffen: 29. Nov. 2019. 
Louis, K. S., Dretzke, B., \& Wahlstrom, K. (2010b). How does leadership affect student achievement? Results from a national US survey. School Effectiveness and School Improvement, 21(3), 315-336. https://doi.org/10.1080/09243453.2010.486586.

Lüdtke, O., Marsh, H.W., Robitzsch, A., \& Trautwein, U. (2011). A $2 \times 2$ taxonomy of multilevel latent contextual models: accuracy-bias trade-offs in full and partial error correction models. Psychological methods, 16(4), 444-467. https://doi.org/10.1037/a0024376.

Ma, X., \& Marion, R. (2019). Exploring how instructional leadership affects teacher efficacy: a multilevel analysis. Educational Management Administration \& Leadership. https://doi.org/10.1177/ 1741143219888742.

Maag Merki, K. (2017). School Improvement Capacity als ein Forschungsfeld der Schulentwicklungsund Schuleffektivitätsforschung - Theoretische und methodische Herausforderungen. In U. Steffens, K. Maag Merki \& H. Fend (Hrsg.), Schulgestaltung - Aktuelle Befunde und Perspektiven der Schulqualitäts- und Schulentwicklungsforschung (S. 269-286). Münster: Waxmann.

MacBeath, J. (2007). Leading learning and learning to lead. Journal für Schulentwicklung, 1(2007), 21-31.

Marks, H.M., \& Printy, S.M. (2003). Principal leadership and school performance: an integration of transformational and instructional leadership. Educational Administration Quarterly, 39(3), 370-397. https://doi.org/10.1177/0013161X03253412.

Marsh, H.W., Lüdtke, O., Nagengast, B., Trautwein, U., Morin, A.J.S., Abduljabbar, A.S., \& Köller, O. (2012). Classroom climate and contextual effects: conceptual and methodological issues in the evaluation of group-level effects. Educational Psychologist, 47(2), 106-124. https://doi.org/10.1080/ 00461520.2012 .670488 .

Moral, C., Martín-Romera, A., Martínez-Valdivia, E., \& Olmo-Extremera, M. (2018). Successful secondary school principalship in disadvantaged contexts from a leadership for learning perspective. School Leadership \& Management, 38(1), 32-52. https://doi.org/10.1080/13632434.2017.1358161.

Morgan, S.L., \& Winship, C. (2015). Counterfactuals and causal inference: methods and principles for social research (2. Aufl.). New York: Cambridge University Press.

Muijs, D. (2011). Researching leadership: towards a new paradigm. In T. Townsend \& J. MacBeath (Hrsg.), Springer international handbook of leadership for learning (Bd. 25, S. 115-125). https://doi.org/10. 1007/978-94-007-1350-5_9.

Mulford, B. (2003). School leaders: Changing roles and impact on teacher and school effectiveness. Education and Training Policy Division, OECD. http://78.41.128.130/dataoecd/61/61/2635399.pdf. Zugegriffen: 29. Nov. 2019.

Murphy, J., Elliott, S. N., Goldring, E., \& Porter, A.C. (2007). Leadership for learning: a research-based model and taxonomy of behaviors. School Leadership \& Management, 27(2), 179-201. https://doi. org/10.1080/13632430701237420.

Muslic, B., Ramsteck, C., \& Kuper, H. (2013). Das Verhältnis von Schulleitung und Schulaufsicht im Kontext testbasierter Schulreform - Kontrastive Fallstudien zur Rezeption von Lernstandsergebnissen im Mehrebenensystem der Schule. In I. van Ackeren, M. Heinrich \& F. Thiel (Hrsg.), Evidenzbasierte Steuerung im Bildungssystem?: Befunde aus dem BMBF-SteBis-Verbund (S. 97-120). https://contentselect.com/media/moz_viewer/536a348d-3aac-4a67-94 f8-20f82efc1343.

OECD (2009). Creating effective teaching and learning environments: first results from TALIS. Paris: OECD.

OECD (2010). TALIS 2008. Technical report. Paris: OECD.

OECD (2016). School leadership for learning. Paris: OECD.

Ogawa, R. T., \& Bossert, S. T. (1995). Leadership as an organizational quality. Educational Administration Quarterly, 31(2), 224-243. https://doi.org/10.1177/0013161X95031002004.

Opdenakker, M., \& Damme, J.V. (2007). Do school context, student composition and school leadership affect school practice and outcomes in secondary education? British Educational Research Journal, 33(2), 179-206. https://doi.org/10.1080/01411920701208233.

Paletta, A., Alivernini, F., \& Manganelli, S. (2017). Leadership for learning: the relationships between school context, principal leadership and mediating variables. International Journal of Educational Management, 31(2), 98-117. https://doi.org/10.1108/IJEM-11-2015-0152.

Pietsch, M., \& Leist, S. (2019). The effects of competition in local schooling markets on leadership for learning. Zeitschrift Für Bildungsforschung, 9(1), 109-134. https://doi.org/10.1007/s35834-0180224-9.

Pietsch, M., \& Tulowitzki, P. (2017). Disentangling school leadership and its ties to instructional practices-An empirical comparison of various leadership styles. School Effectiveness and School Improvement, 28(4), 629-649. https://doi.org/10.1080/09243453.2017.1363787. 
Pietsch, M., Lücken, M., Thonke, F., Klitsche, S., \& Musekamp, F. (2016). Der Zusammenhang von Schulleitungshandeln, Unterrichtsgestaltung und Lernerfolg: Eine argumentbasierte Validierung zur Interpretier- und Nutzbarkeit von Schulinspektionsergebnissen im Bereich Führung von Schulen. Zeitschrift für Erziehungswissenschaft, 19(3), 527-555. https://doi.org/10.1007/s11618-016-0692-4.

Pietsch, M., Scholand, B., Graw, S., Hengstmann, E., \& Kulin, S. (2013). Skalenhandbuch der Schulinspektion hamburg. Fragebögen für Pädagoginnen und Pädagogen, Eltern und Schülerinnen und Schüler [Scale handbook of the hamburg school inspection: questionnaires for teachers, parents and students]. Hamburg: Institut für Bildungsmonitoring und Qualitätsentwicklung.

Pietsch, M., Tulowitzki, P., \& Hartig, J. (2019a). Examining the effect of principal turnover on teaching quality: A study on organizational change with repeated classroom observations. School Effectiveness and School Improvement. https://doi.org/10.1080/09243453.2019.1672759.

Pietsch, M., Tulowitzki, P., \& Koch, T. (2019b). On the differential and shared effects of leadership for learning on teachers' organizational commitment and job satisfaction: a multilevel perspective. $E d u$ cation Administration Quarterly, 55(5), 705-741. https://doi.org/10.1177/0013161X18806346.

Pont, B., Nusche, D., \& Moorman, H. (2008). Policy and practice (Improving school leadership, Bd. 1). Paris: OECD.

Porter, A.C., Polikoff, M.S., Goldring, E.B., Murphy, J., Elliott, S. N., \& May, H. (2010). Investigating the validity and reliability of the Vanderbilt assessment of leadership in education. The Elementary School Journal, 111(2), 282-313. https://doi.org/10.1086/656301.

Preacher, K. J., Zyphur, M.J., \& Zhang, Z. (2010). A general multilevel SEM framework for assessing multilevel mediation. Psychological methods, 15(3), 209-233. https://doi.org/10.1037/a0020141.

Printy, S. M., \& Marks, H.M. (2006). Shared leadership for teacher and student learning. Theory Into Practice, 45(2), 125-132. https://doi.org/10.1207/s15430421tip4502_4.

Ramsteck, C., Muslic, B., Graf, T., Maier, U., \& Kuper, H. (2015). Data-based school improvement. The role of principals and school supervisory authorities within the context of low-stakes mandatory proficiency testing in four German states. The international journal of educational management, 29(6), 766-789.

Reichardt, C.S. (2000). A typology of strategies for rulint out threats to validity. In L. Bickman (Hrsg.), Research design: Donald Campbell's legacy (S. 89-115). Thousand Oaks: SAGE.

Reichardt, C.S. (2011). Evaluating methods for estimating program effects. American Journal of Evaluation, 32(2), 246-272. https://doi.org/10.1177/1098214011398954.

Robinson, V.M.J., Hohepa, M. K., \& Lloyd, C. (2009). School leadership and student outcomes: Identifying what works and why: best evidence synthesis iteration. Wellington: New Zealand Ministry of Education.

Robinson, V. M. J., Lloyd, C. A., \& Rowe, K. J. (2008). The impact of leadership on student outcomes: an analysis of the differential effects of leadership types. Educational Administration Quarterly, 44(5), 635-674. https://doi.org/10.1177/0013161X08321509.

Rogers, P.J. (2008). Using programme theory to evaluate complicated and complex aspects of interventions. Evaluation, 14(1), 29-48. https://doi.org/10.1177/1356389007084674.

Roggenbuck-Jagau, I. (2005). Berufsverständnis und Professionalisierung von Schulleitern. Wiesbaden: VS.

Rowan, B., Raudenbush, S., \& Kang, S. (1991). Organizational design in high schools: a multilevel analysis. American Journal of Education, 99(2), 238-266. https://doi.org/10.1086/443980.

Scheerens, J. (Hrsg.). (2012). School leadership effects revisited-review and meta-analysis of empirical studies. Dordrecht: Springer.

Schmich, J., \& Schreiner, C. (Hrsg.). (2010). Talis 2008: Schule als Lernumfeld und Arbeitsplatz: Vertiefende Analysen aus österreichischer Perspektive. Graz: Leykam.

Schwarz, A., \& Brauckmann, S. (2015). Between facts and perceptions: The area close to school as a context factor in school leadership. Schumpeter Discussion Papers. http://nbn-resolving.de/urn:nbn:de: hbz:468-20150416-103442-7. Zugegriffen: 29. Nov. 2019.

Scriven, M. (1994). The fine line between evaluation and explanation. Evaluation Practice, 15(1), 75-77. https://doi.org/10.1177/109821409401500108.

Scriven, M. (2008). A summative evaluation of RCT methodology: \& an alternative approach to causal research. Journal of MultiDisciplinary Evaluation, 5(9), 11-24.

Scriven, M. (2009). Demythologizing causation and evidence. In S. Donaldson, C. Christie \& M. Mark (Hrsg.), What counts as credible evidence in applied research and evaluation practice? (S. 134-152). https://doi.org/10.4135/9781412995634.d14.

Shamir, B. (2011). Leadership takes time: Some implications of (not) taking time seriously in leadership research. The Leadership Quarterly, 22(2), 307-315. https://doi.org/10.1016/j.leaqua.2011.02.006. 
Shatzer, R.H., Caldarella, P., Hallam, P.R., \& Brown, B.L. (2014). Comparing the effects of instructional and transformational leadership on student achievement: implications for practice. Educational Management Administration \& Leadership, 42(4), 445-459. https://doi.org/10.1177/ 1741143213502192.

Sinharay, S. (2010). How often do subscores have added value? Results from operational and simulated data. Journal of Educational Measurement, 47(2), 150-174. https://doi.org/10.1111/j.1745-3984.2010. 00106.x.

Spillane, J.P. (2006). Distributed leadership. San Francisco: Jossey Bass.

Stentz, J.E., Plano Clark, V.L., \& Matkin, G. S. (2012). Applying mixed methods to leadership research: a review of current practices. The Leadership Quarterly, 23(6), 1173-1183. https://doi.org/10.1016/ j.leaqua.2012.10.001.

Supovitz, J., Sirinides, P., \& May, H. (2010). How principals and peers influence teaching and learning. Education Administration Quarterly, 46(1), 31-56.

Swaffield, S., \& MacBeath, J. (2009). Leadership for learning. In J. MacBeath \& N. Dempster (Hrsg.), Connecting leadership and learning: principles for practice (S. 32-52). London: Routledge.

Teddlie, C. (2005). Methodological issues related to causal studies of leadership: a mixed methods perspective from the USA. Educational Management Administration \& Leadership, 33(2), 211-227. https:// doi.org/10.1177/1741143205051054.

Thompson, G., \& Glas $\varnothing$, L. (2015). Situational leadership theory: a test from three perspectives. Leadership \& Organization Development Journal, 36(5), 527-544. https://doi.org/10.1108/LODJ-10-20130130 .

Thoonen, E.E. J., Sleegers, P. J.C., Oort, F. J., Peetsma, T.T.D., \& Geijsel, F.P. (2011). How to improve teaching practices: the role of teacher motivation, organizational factors, and leadership practices. Educational Administration Quarterly, 47(3), 496-536. https://doi.org/10.1177/0013161X11400185.

Tian, M., Risku, M., \& Collin, K. (2016). A meta-analysis of distributed leadership from 2002 to 2013 : theory development, empirical evidence and future research focus. Educational Management Administration \& Leadership, 44(1), 146-164. https://doi.org/10.1177/1741143214558576.

Timperley, H., \& Robertson, J. (2011). Establishing platforms for leadership and learning. In J. Robertson \& H. Timperley (Hrsg.), Leadership and learning (S. 3-12). Thousand Oaks: SAGE.

Townsend, T., \& MacBeath, J. (Hrsg.). (2011). International handbook of leadership for learning. Dordrecht: Springer.

Townsend, T., Acker-Hocevar, M., Ballenger, J., \& Place, A. W. (2013). Voices from the field: what have we learned about instructional leadership? Leadership and Policy in Schools, 12(1), 12-40. https:// doi.org/10.1080/15700763.2013.766349.

Tulowitzki, P. (2019). Supporting instructional leadership and school improvement? Reflections on school supervision from a German perspective. Journal of Educational Administration, 57(5), 571-581. https://doi.org/10.1108/JEA-03-2019-0040.

Tulowitzki, P., Hinzen, I., \& Roller, M. (2019). Die Qualifizierung von Schulleiterinnen und Schulleitern in Deutschland - ein bundesweiter Überblick. Die Deutsche Schule, 111(2), 149-170. https://doi.org/ 10.31244/dds.2019.02.04.

Tulowitzki, P., Pietsch, M., \& Spillane, J.P. (2020). Leadership for learning. In S. Jornitz \& A. Wilmers (Hrsg.), International perspectives on school settings, education policy and digital strategies. A transatlantic discourse in education research. Opladen: Barbara Budrich.

VanderWeele, T. J. (2015). Explanation in causal inference. New York: Oxford University Press.

Vecchio, R.P. (1987). Situational leadership theory: an examination of a prescriptive theory. Journal of Applied Psychology, 72(3), 444-451.

Vermunt, J.K. (2003). Multilevel latent class models. Sociological Methodology, 33(1), 213-239. https:// doi.org/10.1111/j.0081-1750.2003.t01-1-00131.x.

Wahlstrom, K.L., \& Louis, K. S. (2008). How teachers experience principal leadership: the roles of professional community, trust, efficacy, and shared responsibility. Educational Administration Quarterly, 44(4), 458-495. https://doi.org/10.1177/0013161X08321502.

Warwas, J. (2012). Berufliches Selbstverständnis, Beanspruchung und Bewältigung in der Schulleitung. Wiesbaden: VS.

Wegge, J., \& von Rosenstiel, L. (2004). Führung. In H. Schuler (Hrsg.), Lehrbuch Organisationspsychologie (3. Aufl., S. 475-513). Bern: Huber.

Witziers, B., Bosker, R. J., \& Krüger, M.L. (2003). Educational leadership and student achievement: the elusive search for an association. Educational Administration Quarterly, 39(3), 398-425. https://doi. org/10.1177/0013161X03253411. 
Yammarino, F., \& Dansereau, F. (2008). Multi-level nature of and multi-level approaches to leadership. Leadership Quarterly, 19(2), 135-141. https://doi.org/10.1016/j.leaqua.2008.01.001.

Yammarino, F., \& Dionne, S. (2018). Leadership and levels of analysis: improving leadership research and practice. In R.E. Reggio (Hrsg.), What's wrong with leadership? (S. 41-57). https://doi.org/10.4324/ 9781315163604-3.

Zumbo, B. D., Liu, Y., Wu, A. D., Shear, B. R., Olvera Astivia, O.L., \& Ark, T. K. (2015). A methodology for Zumbo's third generation DIF analyses and the ecology of item responding. Language Assessment Quarterly, 12(1), 136-151. https://doi.org/10.1080/15434303.2014.972559. 